\title{
Flatland Electrons in High Magnetic Fields
}

\author{
M. Shayegan \\ Department of Electrical Engineering \\ Princeton University, Princeton, New Jersey USA
}

November 13, 2018

\section{Contents}

1 Introduction

2 Samples and Measurements 4

2.1 Two-dimensional electrons at the GaAs/AlGaAs interface . . . . . . . . . 4

2.2 Magnetotransport measurement techniques . . . . . . . . . . 8

3 Ground states of the 2D system in a strong magnetic field 8

3.1 The integral quantum Hall effect (IQHE) . . . . . . . . . . . . 8

3.2 Electron-electron interaction and the fractional quantum Hall effect (FQHE) 10

3.3 Composite Fermions . . . . . . . . . . . . . . . . . 12

3.4 The Wigner crystal state . . . . . . . . . . . . . . . . . . 15

3.5 Ferromagnetic state at $\nu=1$ and Skyrmions . . . . . . . . . . . . 17

3.6 Excited Landau levels: competition between uniform- and modulated-density many-body states . . . . . . . . . . . . . . . . 19

3.7 Radiation-induced "zero-resistance" states at low fields . . . . . . . . . . . 22

4 Correlated bilayer electron states 23

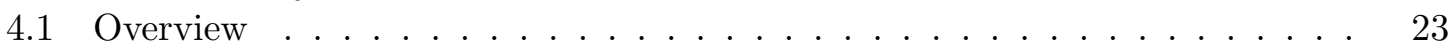

4.2 Even-denominator quantum Hall states in bilayer systems . . . . . . . . 24 24

4.3 The bilayer QHE at $\nu=1$ : electron-hole pairing and Bose-Einstein conden-

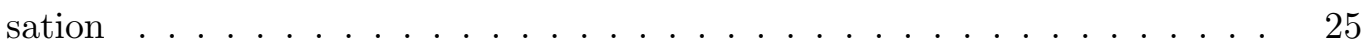

4.4 Insulating phases in bilayer systems: evidence for a bilayer Wigner crystal . 27

5 Summary and future perspectives

6 Acknowledgments $\quad 29$

7 References 


\section{Introduction}

Electrons in a "flatland" are amazing! A simple low-temperature measurement of the resistance of a two-dimensional electron system (2DES) as a function of perpendicular magnetic field $(B)$ reveals why (Fig. 1). In this figure the resistivities along $\left(\rho_{x x}\right)$ and perpendicular $\left(\rho_{x y}\right)$ to the direction of current are shown, and the vertical markings denote the Landau-level filling factor $(\nu)$. Look how the behavior of $\rho_{x x}$ with temperature $(T)$, shown schematically in the inset, changes as a function of the magnetic field. At certain fields, marked $\mathbf{A}, \rho_{x x}$ drops exponentially with decreasing temperature and approaches zero as $T \rightarrow 0$. This is the quantum Hall effect (QHE) and, as you can see in the other trace of Fig. 1, the Hall resistance $\left(\rho_{x y}\right)$ becomes quantized near these fields. The QHE is best described as an incompressible quantum liquid which can possess a high degree of shortrange electron correlation (e.g., when the QHE occurs at a fractional $\nu$ ). Next, look at the $T$-dependence of $\rho_{x x}$ at the fields marked $\mathbf{B}$ (near 13 and $14 \mathrm{~T}$ for this sample). Here $\rho_{x x}$ exponentially increases with decreasing $T$, signaling an insulating behavior. The nature of this insulating state is not entirely clear, but it is generally believed that it is a pinned Wigner solid, a "crystal" of electrons with long-range positional order (see Fig. 2). Now look at what happens at the magnetic field marked C. At this field, $\rho_{x x}$ shows a nearly temperature-independent behavior, reminiscent of a metal. It turns out that at this particular field there are two flux quanta per each electron. The electron magically combines with the two flux quanta and forms the celebrated "composite Fermion," a quasiparticle which now moves around in the 2D plane as if no external magnetic field was applied!

So in one sweep, just changing the magnetic field, the 2DES shows a variety of ground states ranging from insulating to metallic to a "superconducting-like" phase. But wait, that's not all! During the past decade, yet more new phases and phenomena have been discovered (see Figs. 2 and 3). For example, near certain magnetic fields, the spins of electrons have a remarkable texture, as the so-called "Skyrmions" are present. Yet at other fields, the ground state is a "striped phase" where the electron density is modulated in one direction and the electron transport in the plane becomes extremely anisotropic. As it turns out, these ground states are all stabilized primarily by strong electron-electron correlations. The presence of so many novel states attests to the extreme richness of this system, one which has rendered the field of $2 \mathrm{D}$ carrier systems in a high magnetic field among the most active and exciting in solid state physics. It has already led to two physics Nobel prizes, one in 1985 to K. von Klitzing for the discovery of the integral QHE (IQHE) [2], and another in 1998 to R.B. Laughlin, H.L. Stormer, and D.C. Tsui for the fractional QHE (FQHE) 3, 4, but surprises don't seem to stop.

The purpose of this article is to provide a glimpse of some of the exciting experimental results in this field. My presentation will approximately follow the history shown in Fig. 3 and will focus on the following areas:

1. a quick summary of some of the sample parameters and experimental aspects;

2. some basic and general remarks on the different states of a 2DES in a strong perpendicular magnetic field, including the QHE, Wigner crystal, composite Fermions, Skyrmions, and striped phases; 


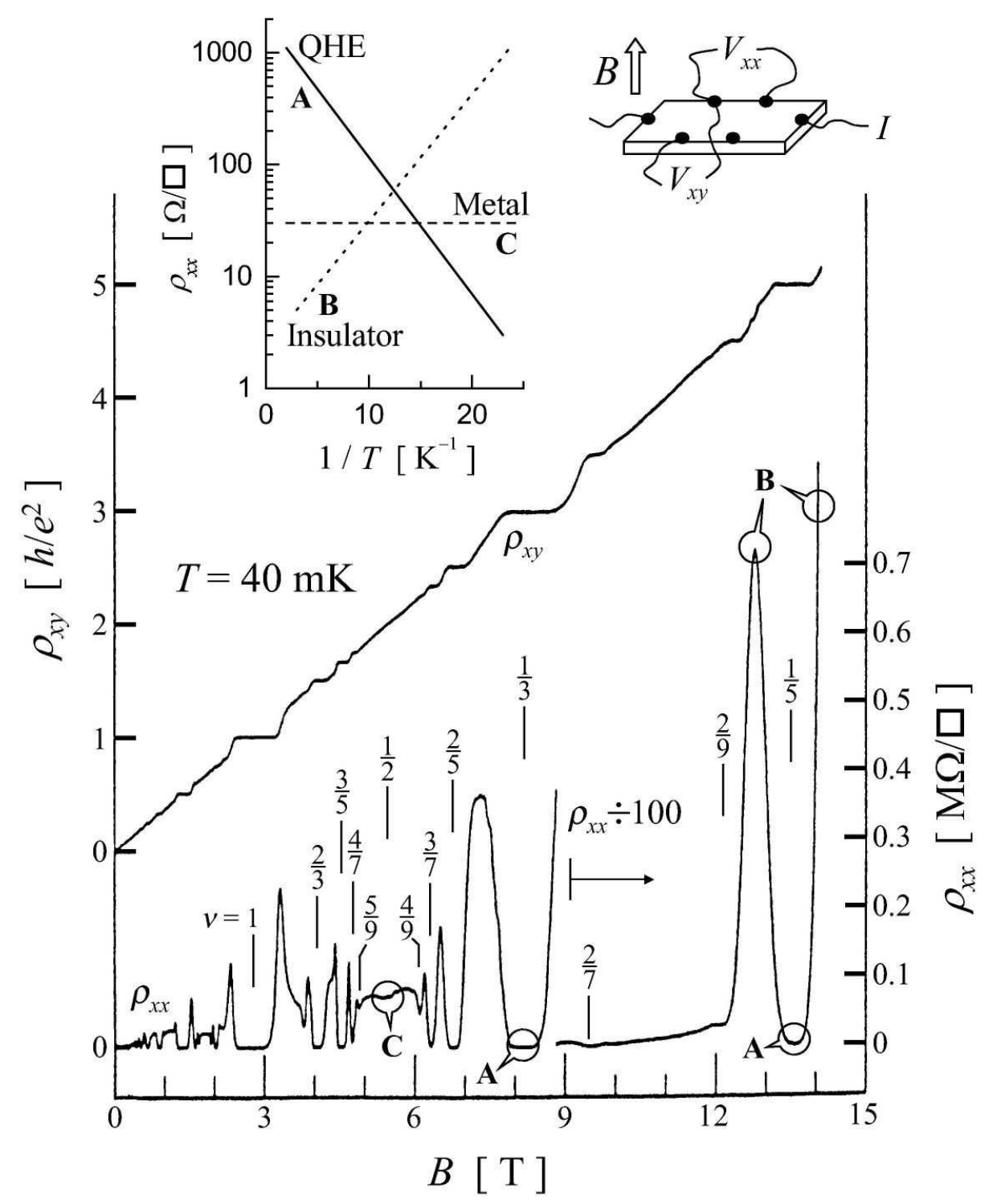

Figure 1: Low-temperature magnetotransport coefficients of a high-quality (low-disorder) 2D electron system in a modulation-doped GaAs/AlGaAs heterostructure with a 2D density of $6.6 \times 10^{10} \mathrm{~cm}^{-2}$. The longitudinal $\left(\rho_{x x}\right)$ and Hall $\left(\rho_{x y}\right)$ resistivities at a temperature of $40 \mathrm{mK}$ are shown in the main figure. The Landau-level filling factors $(\nu)$ are indicated by vertical markings. The right upper inset shows the typical measurement geometry while the left inset schematically illustrates the widely different temperature dependences of $\rho_{x x}$ at different magnetic fields (filling factors), marked by $\mathbf{A}, \mathbf{B}$, and $\mathbf{C}$ in the main figure. (After Sajoto et al. [1].) 


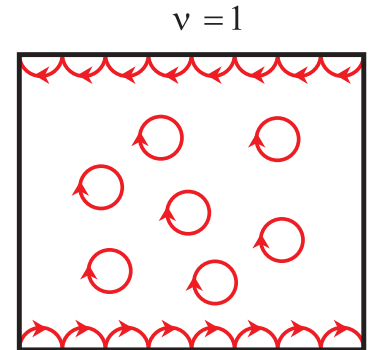

Integer QHE

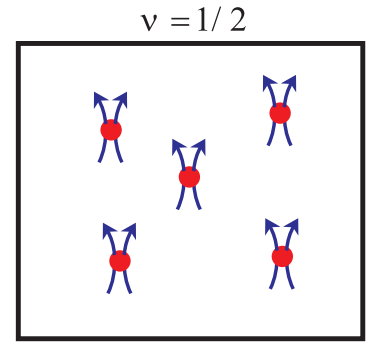

Composite Fermions

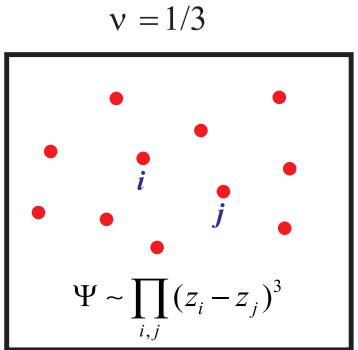

Fractional QHE

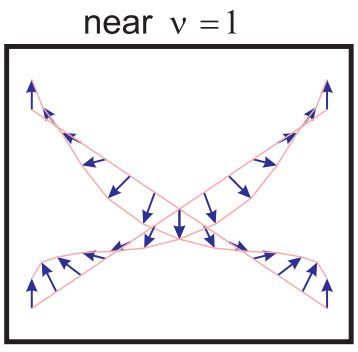

Skyrmions

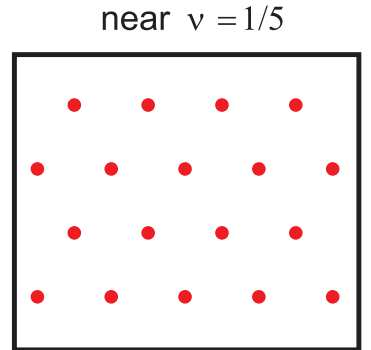

Wigner Crystal

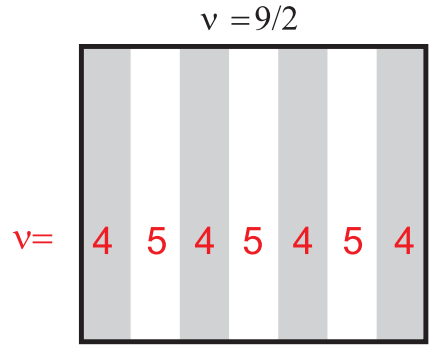

Striped Phases

Figure 2: Some of the different states of a low-disorder 2D electron system in a strong perpendicular magnetic field. The only parameter that is changing is the Landau level filling factor $(\nu)$ which is inversely proportional to the magnetic field. Except for the integer QHE, all the other states are stabilized by the electron-electron interaction.

3. bilayer electron systems in which the additional (layer) degree of freedom leads to unique QHE and insulating states which are stabilized by strong intralayer and interlayer correlations. A highlight is the recent observation of pairing of carriers in two closely-spaced, interacting layers and the signatures of the Bose-Einstein condensation of the pairs (excitons).

I'd like to emphasize that this article is far from properly dealing with all the important and exciting aspects of the physics of $2 \mathrm{D}$ systems in high magnetic fields. It provides only a limited and selective sample of transport measurements. Readers interested in more details are referred to the original papers as well as extensive review articles and books [5, 6, 7, 8, 9, 10. Also, there will be a minimal treatment of theory here; for more details and insight, I suggest reading the illuminating article by D. Yoshioka in this volume and various articles in Refs. 5 to 10 .

\section{Samples and Measurements}

\subsection{Two-dimensional electrons at the GaAs/AlGaAs interface}

One of the simplest ways to place electrons in a flatland is to confine them to the interface between two semiconductors which have different bandgaps. An example is shown in Fig. 4 


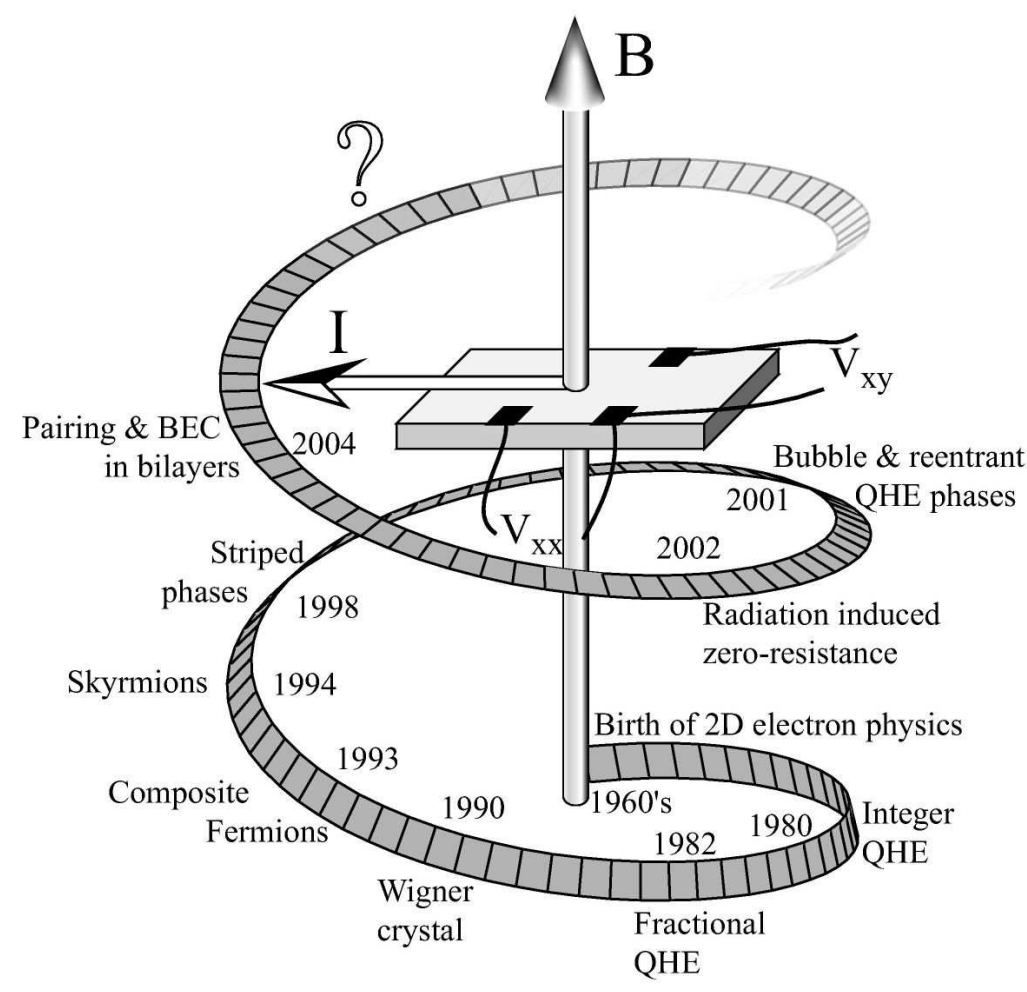

Figure 3: Some of the noteworthy discoveries in the field of flatland electrons in a perpendicular magnetic field.

(left) where a 2DES is formed at the interface between undoped GaAs and AlGaAs [9]. The larger bandgap of AlGaAs leads to its conduction-band energy $\left(E_{C B}\right)$ being higher than GaAs. The system is "modulation-doped" 11] meaning that the dopant atoms (in this case, Si donors) are placed in AlGaAs at some distance away from the interface. The electrons from the donors find it energetically favorable to transfer to the lower energy conductionband of GaAs. But as they transfer, an electric field sets up between the positively-charged (ionized) donors in AlGaAs and the transferred electrons in GaAs. This electric field limits the amount of charge transfer. Figure 4 (lower left) schematically shows $E_{C B}$ as a function of position, at equilibrium, after the charge transfer has taken place [9]. An alternative way to form a 2DES is to confine the electrons in a GaAs quantum well which is flanked by modulation-doped AlGaAs barriers. This is shown schematically in Fig. 4 on the right.

A key point in the structures of Fig. 4 is that the 2DES is separated from the ionized dopants. As a result, the scattering of electrons by the ionized impurity potential is significantly reduced, meaning that the $2 \mathrm{D}$ electrons are essentially "free" to move in the plane. It turns out this is crucial for much of the phenomena observed in these systems: by reducing the disorder and the electron-impurity interaction, electrons are allowed to interact with each other, and the result is a host of new many-body ground and excited states. Another 


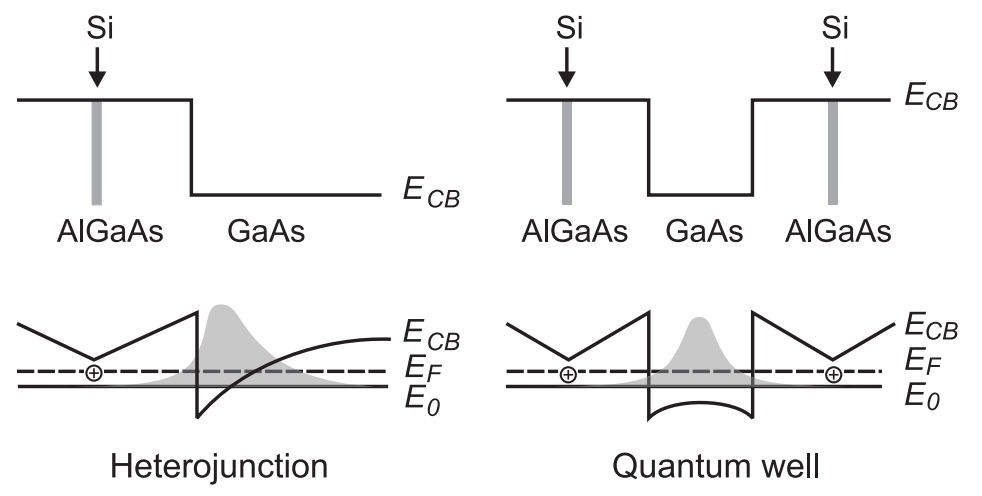

Figure 4: Schematic descriptions of modulation-doped GaAs/AlGaAs samples. Since the conduction-band edge $\left(E_{C B}\right)$ of GaAs lies lower in energy than that of AlGaAs, electrons transfer from the doped AlGaAs region to the undoped GaAs to form a quasi-2D electron system (2DES) at the heterojunction interface between GaAs and AlGaAs (left), or in a GaAs quantum well (right). In both cases, the 2DES is separated from the doped AlGaAs region by an undoped AlGaAs (spacer) layer to minimize electron scattering by the ionized impurities. The ground state subband energy $E_{o}$ and the Fermi level $E_{F}$ are shown. Note that the electron wavefunction, $\psi(z)$, has a finite extent in the direction perpendicular to the plane in which the electrons move freely.

important message here is that although we call the system "two-dimensional," the electron wavefunction $\psi(z)$ spreads in the $z$ direction by a finite amount, typically $\sim 10 \mathrm{~nm}$. This finite layer-thickness plays an important role and should be taken into account when comparing theoretical calculations and experimental results: it distinguishes between "ideal" $2 \mathrm{D}$ system assumed in many calculations and the "real" quasi-2D, experimental system.

How does one fabricate structures like those in Fig. 4 and what are the details of a typical sample structure? The best quality GaAs/AlGaAs samples are presently grown by molecular beam epitaxy (MBE) 12. The MBE system (Fig. 5) is essentially a very "clean" high-vacuum evaporation chamber. A GaAs substrate, heated to about $630^{\circ} \mathrm{C}$, is positioned in front of effusion cells (ovens) each of which contains one of the required elements (Ga, $\mathrm{Al}, \mathrm{As}$, and $\mathrm{Si}$ ). The ovens are heated to appropriate temperatures to produce fluxes of these elements which can impinge on the GaAs substrate. Each oven also has a shutter which is controlled, normally via a computer, to produce the desired structure. Under these circumstances, and with a growth rate of about one monolayer of GaAs per second (which is roughly $1 \mu \mathrm{m} /$ hour), one can grow very high quality, single-crystal structures with nearly any design.

What determines the "quality" of the 2DES? For the electron-interaction-dominated phenomena in which we are interested here, the best sample is typically one with the least amount of imperfections such as interface irregularities, ionized impurities, etc. It is this consideration that leads to structures where the 2DES is typically separated from the $\mathrm{Si}$ dopants by a very thick spacer layer of undoped AlGaAs. Details and rationale for other fabrication procedures such as growth interruptions, the use of a spacer with graded $\mathrm{Al}$ 


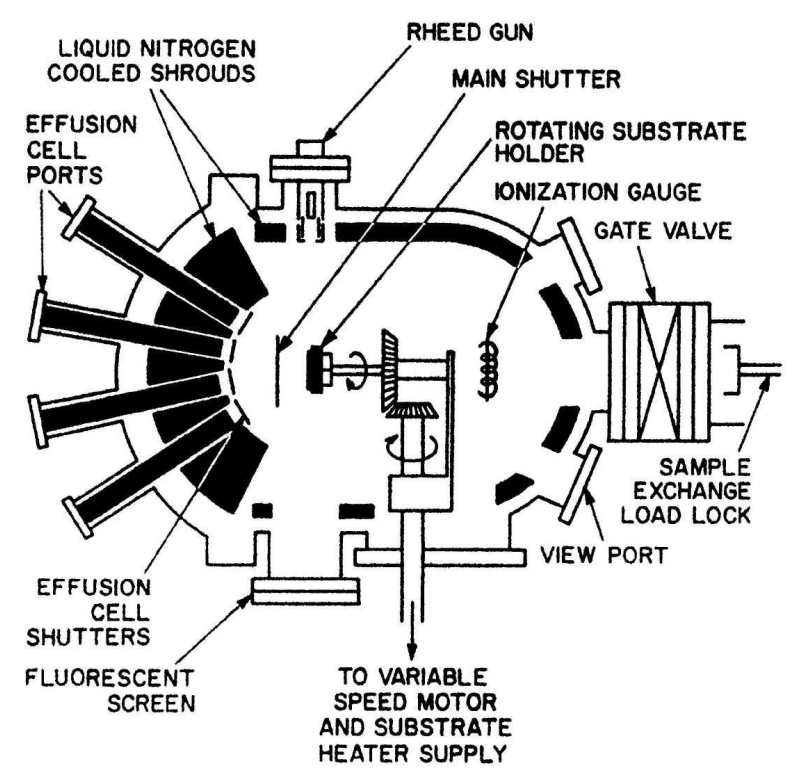

Figure 5: Cross-sectional view of a molecular beam epitaxy (MBE) growth chamber (after Cho [12]), essentially a very high-vacuum evaporation chamber with a base pressure of $10^{-14}$ atmosphere. The chamber is equipped with various vacuum pumps, such as ion-pumps and cryopumps, and also can have analytical equipment such as a reflection high-energy electron diffractometer (RHEED) to monitor in-situ the substrate surface morphology as well as growth rate.

composition, double- $\delta$-doping etc., can be found in Ref. 13. But a very important factor determining the quality of the 2DES, one which is not explicitly apparent in the structures of Fig. 4, is the amount of residual (or unintentional) impurities that are incorporated throughout the structure during the MBE growth. These impurities are always present because the vacuum in the MBE chamber is not perfect, and also because the source materials (Ga, Al, etc.) used in the ovens are not $100 \%$ pure. It turns out in fact that in structures with a large $(>200 \mathrm{~nm})$ spacer layer thickness, the most important factor in obtaining very low-disorder 2DES is the purity of the grown material and not the specific details of the structural parameters. The vacuum integrity of the MBE growth chamber and the cleanliness and purity of the source materials and the GaAs substrate are therefore of paramount importance for the fabrication of state-of-the-art 2DESs.

A measure of the electronic "quality" of a 2DES is its low-temperature mobility, $\mu$. Over the years, the mobility of modulation-doped GaAs/AlGaAs heterostructures has improved tremendously and the record stands at over $10^{7} \mathrm{~cm}^{2} / \mathrm{Vs}$ for a 2DES density $(n)$ of $\sim 2 \times 10^{11}$ $\mathrm{cm}^{-2}$, implying a mean-free-path of tens of microns [14, 15]. This mobility is more than $\sim 10^{4}$ times higher than $\mu$ for a uniformly-doped GaAs layer, demonstrating the striking power of modulation-doping. As mentioned in the last paragraph, the mobility in such thickspacer structures is in fact limited by the concentration of the non-intentional (residual) 
impurities. This is evidenced by the observation [13, 16, 17] that $\mu \sim n^{\gamma}$ with $\gamma \simeq 0.6$; this is the dependence expected if the dominant source of scattering is the residual impurities in the close proximity of the 2DES [18. The residual impurity concentration, deduced from the mobility values for state-of-the-art 2DES with $\mu \gtrsim 10^{7} \mathrm{~cm}^{2} / \mathrm{Vs}$ for $n \gtrsim 5 \times 10^{10} \mathrm{~cm}^{-2}$ is $n_{i} \lesssim 1 \times 10^{13} \mathrm{~cm}^{-3}$, consistent with the residual GaAs doping expected in very clean MBE systems. An $n_{i} \sim 10^{13} \mathrm{~cm}^{-3}$ means that the average distance between the residual impurities $\sim 500 \mathrm{~nm}$ is much larger than the typical inter-electron distance in the 2DES $\left(\sim 45 \mathrm{~nm}\right.$ for $\left.n=5 \times 10^{10} \mathrm{~cm}^{-2}\right)$. Clearly in such low-disorder $2 \mathrm{D}$ systems it is reasonable to expect that the physics can be dominated by electron-electron interaction.

\subsection{Magnetotransport measurement techniques}

A variety of experimental techniques has been used to probe the electrical, optical, thermal, and other properties of the 2DES in a high magnetic field. The bulk of the measurements, however, have been of the magnetotransport properties. Magnetotransport measurements are also by far the main topic of this paper. I therefore briefly discuss such measurements here. In typical $d c$ (or low-frequency, $\lesssim 100 \mathrm{~Hz}$ ) transport experiments, the diagonal and Hall resistivities are measured in a Hall bar or van der Pauw geometry with $\sim 1 \mathrm{~mm}$ distance between the contacts. Contacts to the $2 \mathrm{DES}$ are made by alloying In or InSn in a reducing atmosphere at $\sim 450^{\circ} \mathrm{C}$ for about 10 minutes. High-frequency measurements often involve more specialized geometries and contacting schemes. The low-temperature 2D carrier concentration can be varied by either illuminating the sample with a light-emitting diode or applying voltage (with respect to the $2 \mathrm{DES}$ ) to a back- and/or front-gate electrode. Low temperatures are achieved using a ${ }^{3} \mathrm{He} /{ }^{4} \mathrm{He}$ dilution refrigerator, while the magnetic field is provided either by a superconducting solenoid or a Bitter magnet, or a combination of the two. The low-frequency magnetotransport measurements are typically performed with a current excitation of $\lesssim 10^{-9} \mathrm{~A}$, corresponding to an electric field of $\lesssim 10^{-4} \mathrm{Vcm}^{-1}$, and using the lock-in technique.

\section{Ground states of the 2D system in a strong magnetic field}

\subsection{The integral quantum Hall effect (IQHE)}

A large magnetic field applied perpendicular to the plane of a 2DES acts like a harmonic oscillator potential and leads to the quantization of the orbital motion. The allowed energies are quantized and are given by the "Landau Levels" (LLs), $\left(N+\frac{1}{2}\right) \hbar \omega_{c}$, where $N=0,1,2, \ldots$ and $\hbar \omega_{c}=\hbar e B / m^{*}$ is the cyclotron energy. For a system with a finite effective Lande $g$ factor $\left(g^{*}\right)$, the energy spectrum is further quantized as each LL is spin-split to two levels separated by the Zeeman energy $\left|g^{*} \mu_{B} B\right|$ where $\mu_{B}$ is the Bohr magneton. This evolution of the density-of-states, $D(E)$, for a $2 \mathrm{D}$ system in a magnetic field is schematically shown in Fig. 6. Note that for $2 \mathrm{D}$ electrons in GaAs, $m^{*}=0.067 m_{o}$ and $g^{*} \simeq-0.44$, so that the cyclotron energy is about 70 times larger than the (bare) Zeeman energy.

The degeneracy of each spin-split quantized energy level is $e B / h$. Since this degeneracy increases with $B$, to keep the total $2 \mathrm{D}$ density of the system constant, the Fermi energy 


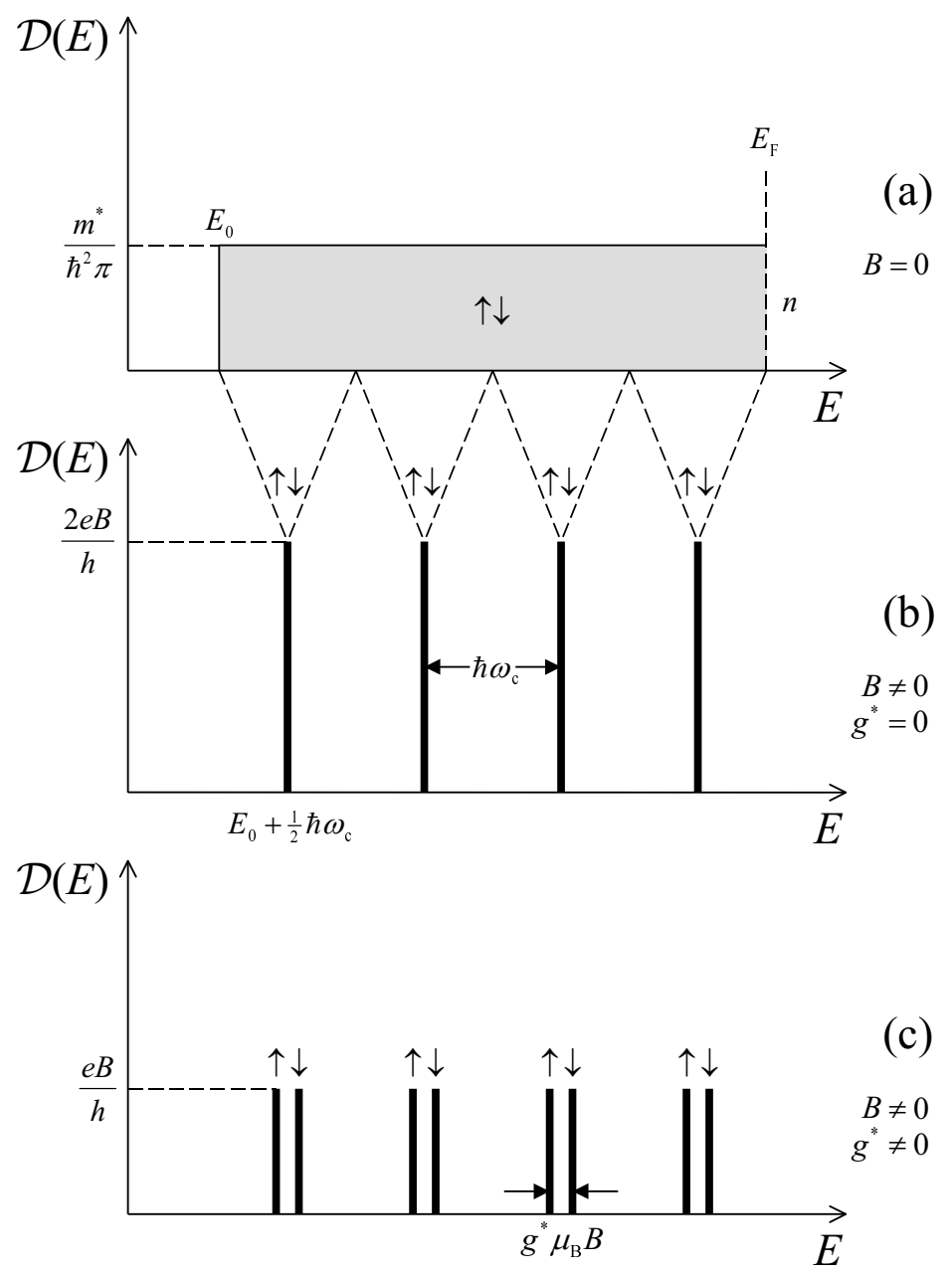

Figure 6: Density-of-states as a function of energy for a 2D carrier system: (a) in the absence of a magnetic field, (b) with a magnetic field $(B)$ applied perpendicular to the 2D plane but neglecting the spin-splitting of the resulting Landau levels, and (c) with spinsplitting included. As is typical for a $2 \mathrm{D}$ electron system in a standard, single GaAs/AlGaAs heterojunction, here it is assumed that only one (size-quantized) electric subband, whose edge energy is marked by $E_{o}$, is occupied.

$\left(E_{F}\right)$ has to move so that fewer and fewer LLs are occupied with increasing $B$. The number of spin-split LLs occupied at a given $B$ is defined as the filling factor and is given by $\nu=$ $n /(e B / h)=n h / e B$. Equivalently, $\nu$ is the number of electrons per flux quantum $\Phi_{o}=h / e$. As $B$ is increased and $E_{F}$ passes through the oscillating $D(E)$, nearly all properties of the system, such as electrical resistivity, magnetic susceptibility, heat capacity, etc., oscillate. The magnetoresistance oscillations are often called Shubnikov-de Haas oscillations. The oscillations are periodic in $1 / B$ with frequency $n h / e$ or $n h / 2 e$, depending on whether or not the spin-splitting is resolved. This means that from a measurement of the frequency of the oscillations one can deduce the density.

The delta-function-like energy levels shown in Fig. 6 are for an ideally pure 2DES. In the presence of disorder, the levels are broadened with their width, $\Gamma$, being of the order of $\hbar / \tau_{q}$ 
where $\tau_{q}$ is the quantum lifetime of the carriers. The states in the LLs' tails are localized and only the centers of the LLs contain current-carrying extended states. Now suppose the filling factor is $i$, or nearly $i$, so that $E_{F}$ lies in the localized states between the $i$ and $(i+1)$ LL. If the disorder and temperature are sufficiently small so that $\Gamma$ and the thermal energy $\left(k_{B} T\right)$ are both smaller than the LL separation, then as $T \rightarrow 0$ the longitudinal conductivity $\left(\sigma_{x x}\right)$ vanishes and the transverse or Hall conductivity $\left(\sigma_{x y}\right)$ becomes quantized at a value that is equal to $i e^{2} / h$. This is the integral QHE. That $\sigma_{x x} \rightarrow 0$ is simply a consequence of there being no extended states in the bulk of the $2 \mathrm{D}$ system to carry current. There are, however, $i$ current-carrying "edge states" near the edge of the sample (see the top left sketch in Fig. 2) and this leads to $\sigma_{x y}$ being quantized although demonstrating this quantization is more subtle (see, e.g., Refs. 6-8). Note also that, according to the simple relations which convert the elements of the conductivity tensor to those of the resistivity tensor, $\rho_{x x}=\sigma_{x x} /\left(\sigma_{x x}^{2}+\sigma_{x y}^{2}\right)$ and $\rho_{x y}=\sigma_{x y} /\left(\sigma_{x x}^{2}+\sigma_{x y}^{2}\right)$. Therefore, $\sigma_{x x}=0$ and $\sigma_{x y}=i e^{2} / h$ mean that $\rho_{x x}=0$ and $\rho_{x y}=h / i e^{2}$. This explains the experimental result in Fig. 1 for the Hall bar sample shown in the inset.

To summarize, the IQHE is a consequence of: (1) the quantization of the 2D system's energy levels into a set of well-defined (but broadened) LLs with separation greater than $k_{B} T$, and (2) the presence of localized states in between these LLs. Note that no electronelectron interaction is needed to bring about or to explain the IQHE.

\subsection{Electron-electron interaction and the fractional quantum Hall effect (FQHE)}

Suppose $B$ is sufficiently raised so that $\nu<1$. At $T=0$ the kinetic energy of the 2DES is quenched and the system enters a regime where, in the absence of disorder, its ground state is determined entirely by the electron-electron interaction. In the infinite $B$ limit, the system approaches a classical 2D system which is known to be an electron crystal (Wigner crystal) 19. At finite $B$, the electrons cannot be localized to a length smaller than the cyclotron orbit radius of the lowest LL, or the magnetic length $l_{B}=(\hbar / e B)^{1 / 2}=(\nu / 2 \pi n)^{1 / 2}$, and the ground state is typically a gas or liquid. However, when $l_{B}$ is much smaller than the average distance between electrons, i.e. when $\nu \ll 1$, a crystalline state is possible [20. We will return to this crystalline state in Section 3.4.

A competing ground state of the 2D system at high $B$ is the FQHE liquid [3, 4]. Ironically, the work that led to the discovery of the new and totally unexpected FQHE phenomenon [3] was itself one of the early experimental searches for the magnetic-field-induced Wigner crystal! In high-quality, low-disorder 2D carrier systems, in fact, the dominant ground states of the system are the FQHE states (Fig. 7). The FQHE, observed at the principal fillings $\nu=1 / q$ and other rational fractional fillings $\nu=p / q$ ( $q=$ odd integer) is characterized by the vanishing of $\rho_{x x}$ and the quantization of $\rho_{x y}$ at $(q / p)\left(h / e^{2}\right)$ as $T \rightarrow 0$. The effect is phenomenologically similar to the IQHE but its origin is very different. The FQHE state is an intrinsically many-body, incompressible quantum liquid, described by the 
Laughlin wavefunction:

$$
\Psi_{m}^{\nu} \sim \prod_{i, j}\left(z_{i}-z_{j}\right)^{m} \times \exp \left(-\sum_{i} \frac{\left|z_{i}\right|^{2}}{4 l_{B}}\right) .
$$

Here $z_{i}$ and $z_{j}$ are the (complex) coordinates of pairs of electrons in the plane (Fig. 2, top center panel), and $m=1 / \nu$ is an odd integer so that the wavefunction is antisymmetric when two electrons are interchanged (Pauli exclusion principle). Note also that the Coulomb repulsion between electrons is built into this wavefunction as it becomes small when two electrons come close to each other. The wavefunction has strong short-range correlation but it does not describe a crystalline phase as it has no long-range order (see e.g., the "snap shots" shown in Fig. 7.7 of Ref. 6).

The FQHE has many fundamental and interesting characteristics among which I briefly mention three here. First, the incompressibility implies that the ground state is separated from its excitations by a finite energy gap, $\Delta$. Experimentally $\Delta$ can be measured from the activated $T$-dependence of $\rho_{x x}$ according to $\rho_{x x} \sim \exp \left(-\Delta / 2 k_{B} T\right)$. This is superficially similar to the energy gap between the LLs which leads to the IQHE, but the origin of the FQHE gap is entirely many-body. The theoretical $\Delta$ for the $\nu=\frac{1}{3}$ FQHE in an ideal 2DES, with no disorder, zero layer-thickness, and infinitely separated LLs, is $\sim 0.1 e^{2} / 4 \pi \varepsilon l_{B}$, where $\varepsilon$ is the dielectric constant of the host material (GaAs). In real samples, however, the finite-layer thickness, LL mixing, and the ubiquitous disorder lead to a gap which is much smaller than $0.1 e^{2} / 4 \pi \varepsilon l_{B}$ (see, e.g., Ref. 22). Finite layer thickness, for example, leads to a softening of the short-range component of the Coulomb interaction, and results in a weakening of the FQHE. In fact, experiments [23] and calculations 24] have revealed that once the layer thickness exceeds $\sim 3 l_{B}$, the FQHE quickly collapses.

A second, quite intriguing yet fundamental feature of the FQHE is that its elementary excitations carry fractional charge $e^{*}=e / m$. There have been several reports of measuring this fractional charge, one of the latest being measurements of the quantum shot noise which is proportional to the charge of the conducting carriers [25]. In these measurements, the current noise was monitored as a function of the backscattered current which results from the tunneling between the FQHE edge states in a point-contact (constriction). The results near $\nu=\frac{1}{3}$ indeed reveal that the current carrying particles have charge $e / 3$.

The third noteworthy feature is the existence of FQHE states not only at the primary fractional fillings such as $\nu=\frac{1}{3}$ and $\frac{1}{5}$, but also at a host of other odd-denominator fillings. Examples are the states at $\nu=\frac{2}{5}, \frac{3}{7}, \ldots$ and $\nu=\frac{3}{5}, \frac{4}{7}, \ldots$ which can be seen in Figs. 1 and 7 . The strength of these states, namely their measured energy gaps, typically decreases as the denominator of their filling gets larger. Also, they appear to form a sequence of decreasing strength as one goes from the primary state, such as $\nu=\frac{1}{3}$, towards the even-denominator filling $\left(\nu=\frac{1}{2}\right)$ at which there is no FQHE state. We will revisit these observations in Section 3.3. Note also that there are unusual and not yet well-understood FQHE states at even-denominator fillings such as $\nu=\frac{5}{2}$ (see Section 3.6). 


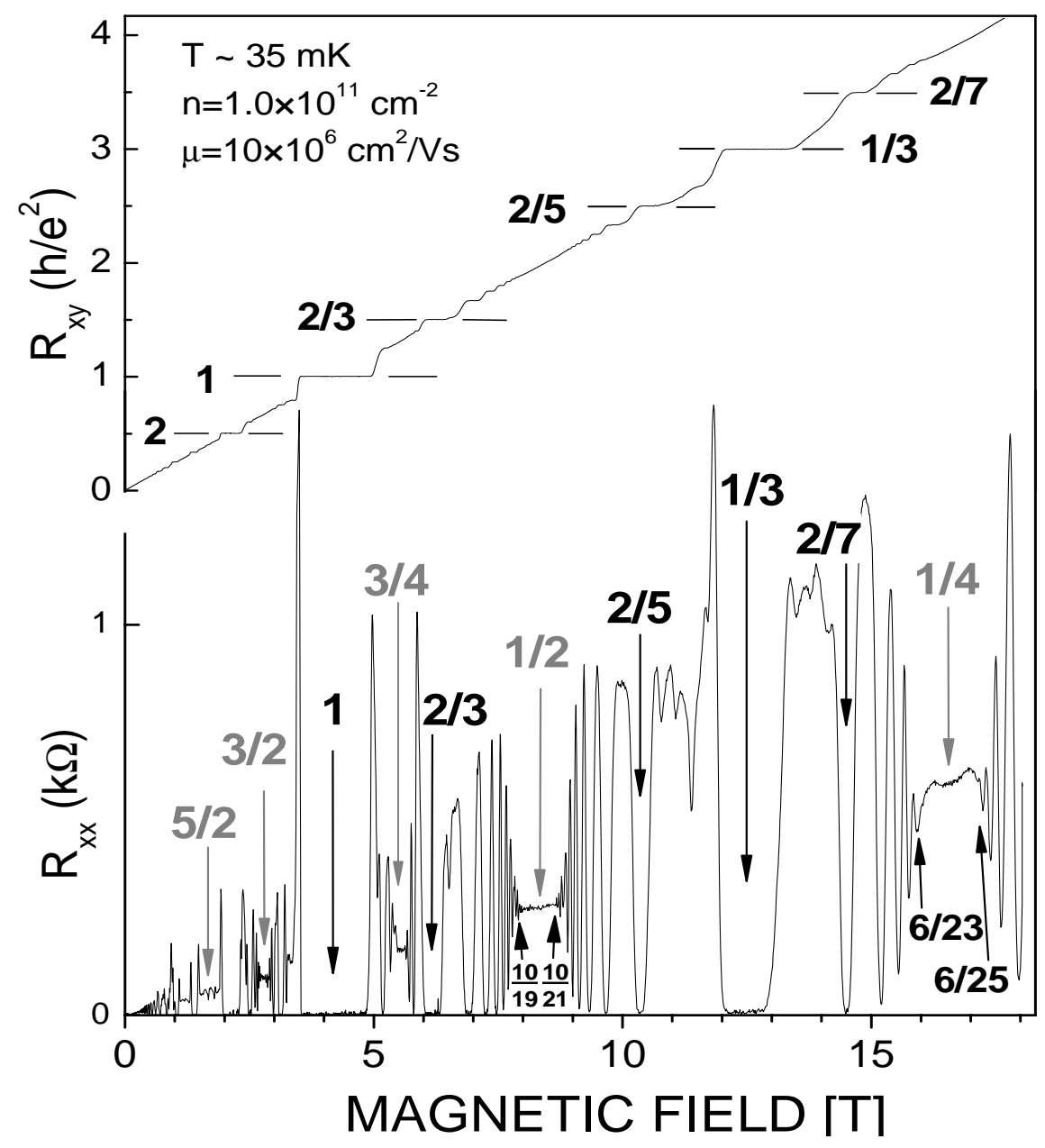

Figure 7: Magnetoresistance traces for a very high quality (mobility about $10^{7} \mathrm{~cm}^{2} / \mathrm{Vs}$ ) GaAs/AlGaAs sample, showing numerous fractional quantum Hall states. (After Pan et al. [21.)

\subsection{Composite Fermions}

The observation that the higher order FQHE states at fillings with increasingly larger denominators have weaker strengths initially led to an explanation for these states based on a "hierarchical" scheme where each state is considered the "parent" state for the adjacent (in filling factor) weaker state. The idea is that as one deviates from the exact filling for a given FQHE state, quasiparticles are created above the energy gap; these quasiparticles then interact and form an incompressible liquid once their density to magnetic flux ratio reaches certain values. Such a hierarchical construction can generate all the odd-denominator fractions, and explains certain features of the observed FQHE sequences. However, it fails to account for the observed strength/weakness of all FQHE states. Also, in this scheme, the wavefunctions of the higher order states turn out to be much more complex than those for 
the primary states. Moreover, such description of the FQHE differs entirely from that of the IQHE while it is hard to overlook the striking similarity between the FQHE sequence, e.g., at $\nu=\frac{1}{3}, \frac{2}{5}, \frac{3}{7} \ldots$ and the IQHE sequence at $\nu=1,2,3, \ldots$ In fact, if we "slide" the $5.5<B<9$ T portion of the magnetoresistance trace in Fig. 1 to the left so that the position of $\nu=\frac{1}{2}$ is now the "zero" of the (effective) magnetic field, we can see a one-to-one correspondence between the above IQHE and FQHE sequences, both in terms of the field positions of $\rho_{x x}$ minima and their relative strength. (This is true if we assume that the 2DES is fully spin-polarized at all fields.)

Such observations prompted the search for a description of the QHE which somehow links the integral and fractional effects. This has culminated in a remarkable description in which an even number of fluxes combine with an electron to form a new, "composite" Fermion $(\mathrm{CF})$ [26]. The electron-electron interaction and the large magnetic field are embedded in this flux-electron quasiparticle so that the system now behaves as if it contains (essentially) non-interacting particles moving in an "effective" magnetic field which is the balance of the external field once the attached fluxes are deducted. For example, focusing on the range $\frac{1}{3} \leq \nu \leq \frac{1}{2}$ and attaching two fluxes to each electron, the CF description maps the FQHE at $\nu=\frac{1}{3}, \frac{2}{5}, \frac{3}{7}, \ldots$ to the IQHE at $\nu=1,2,3, \ldots$ Perhaps even more intriguing is the notion that the effective field at $\nu=\frac{1}{2}$ is zero for the CFs so that, at $\nu=\frac{1}{2}$, they ignore the large external magnetic field and move about as if there is no magnetic field.

More rigorously, a gauge transformation that binds an even number of magnetic flux quanta $\left(2 l \Phi_{0}\right.$ where $l$ is an integer and $\Phi_{0} \equiv h / e$ is the flux quantum) to each electron maps the 2DES at even-denominator fillings to a system of CFs at a vanishing $B_{\text {eff }}[26$. Such transformation elegantly maps a FQHE observed at the 2DES filling $\nu$ to an IQHE for the CF system at filling $\nu^{\prime}$ where $\nu^{\prime}=\nu /(1-2 l \nu)$. In addition, since $B_{\text {eff }}=0$ at $\nu=1 / 2 l$, the CF system should possess certain Fermi-liquid-like properties. Most notably, the CFs should have a Fermi surface at $\nu=\frac{1}{2}$, just like electrons do at zero magnetic field, and should therefore support phenomena such as geometrical resonances and $\mathrm{CF}$ ballistic transport [26].

Here I present, as an example, the results of an experiment which demonstrate the surprisingly simple behavior of CFs near $\nu=\frac{1}{2}$, namely their semiclassical, ballistic motion under the influence of $B_{\text {eff }}$. Figure 8 shows data from a magnetic focusing experiment 27. near $B=0$ (bottom trace) and $\nu=\frac{1}{2}$ (top trace). The geometry of the experiment is sketched in the inset, which shows the top view of the sample. Parts of the sample are etched (thick lines in Fig. 8 inset) so that the 2DES is separated into three regions which are connected by two narrow constrictions (point-contacts). The distance between the two constrictions, $L$, is chosen to be smaller than or of the order of the mean-free-path of the electrons. Ballistic electrons are then injected from the lower-left section to the upper section through the "injector" constriction by passing a current between the ohmic contacts marked $+\mathrm{I}$ and -I. Now a small $B$-field is applied perpendicular to the plane to "bend" the semiclassical, ballistic trajectory of the injected electrons as they travel in the upper section. As $B$ is increased, whenever $L$ matches a multiple integer of the electron's semiclassical cyclotron orbit diameter, $d_{c}=2 m^{*} v_{F} / e B=2 \hbar k_{F} / e B$, the ballistic electrons impinge on the "collector" constriction, either directly or after one or more bounces off the 


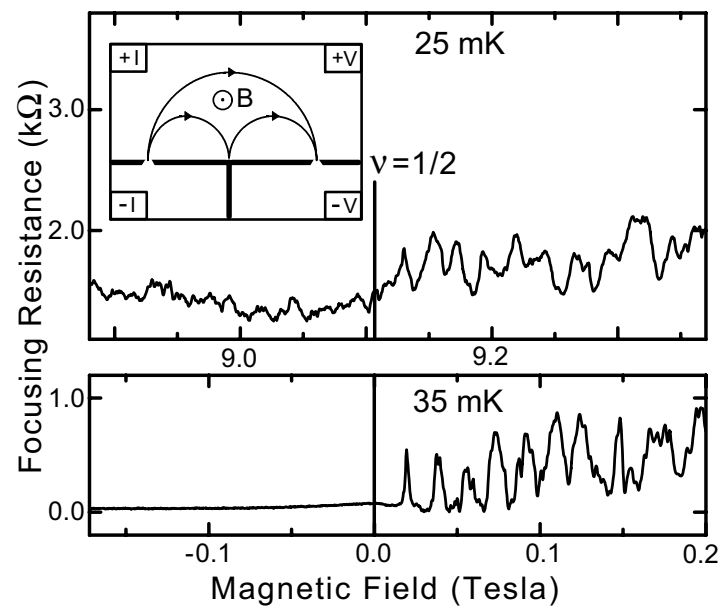

Figure 8: Magnetic focusing spectra are shown for 2D electrons near zero external magnetic field (bottom trace) and for composite Fermions near $\nu=\frac{1}{2}$ (top trace) where the external field is about 9.1 T. In the top trace, the position of $\nu=\frac{1}{2}$ marks the zero of the effective magnetic field $\left(B_{\text {eff }}\right)$ for CFs. Both traces exhibit peaks at fields where the distance between the injector and collector point-contacts ( $L \simeq 5.3 \mu \mathrm{m}$ in this case) matches a multiple integer of the classical cyclotron orbit diameter. The inset schematically shows the top view of the sample. (After Goldman et al. [27.)

focusing barrier separating the two constrictions. At these $B$, one observes a maximum in the voltage measured between the lower-right and the upper sections (contacts marked $+\mathrm{V}$ and $-\mathrm{V})$. The traces shown in Fig. 8 are the voltages measured between contacts $+\mathrm{V}$ and $-\mathrm{V}$, normalized to the current injected between contacts $+\mathrm{I}$ and -I. Maxima can be clearly seen in the lower trace of Fig. 8 for $B>0$ and their positions are indeed consistent with the values of $L$ and $k_{F}$ for this 2DES. Note that for $B<0$, the electrons are deflected to the left and no magnetic focusing is expected, consistent with the absence of any observed maxima.

The experiments of Goldman et al. [27] reveal oscillations of the resistance not only near $B=0$ for electrons, but also near $\nu=\frac{1}{2}$ (upper trace of Fig. 8). The data provide a remarkable demonstration of the "classical", ballistic motion of the CFs under the influence of $B_{\text {eff }}$. Note that $B_{\text {eff }}$ is only a few tenths of a Tesla while the real external magnetic field is about 12 Tesla! The large external magnetic flux felt by the interacting electrons is replaced by the much smaller flux influencing the apparently simple flux-electron composites. The data of Fig. 8 also provide a direct determination of the Fermi wavevector as well as an estimate for the ballistic mean-free-path of the CFs $(\simeq 1 \mu \mathrm{m})$.

As remarkable and perhaps non-intuitive as the CF picture may be, it has received compelling verification through several key experiments [26]. These include measurements of the surface acoustic wave propagation, FQHE activation energies, CF effective mass, resistance oscillations in antidot arrays, magnetic focusing, low- $T$ thermopower, magnetooptics, CF spin, temperature dependence of the CF conductivity at $\nu=\frac{1}{2}$ and $\frac{3}{2}$, and 


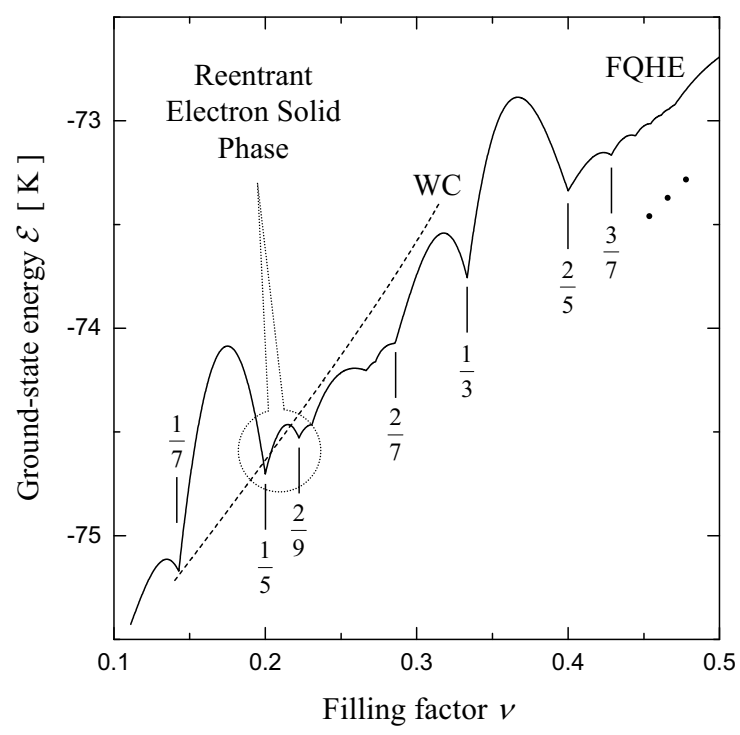

Figure 9: Energies of two competing ground states of a $2 \mathrm{D}$ electron system at high perpendicular magnetic fields. The FQHE incompressible liquid states occur at special odddenominator fillings as the downward energy "cusps" indicate (solid curve). The Wigner crystal (WC) state has monotically decreasing energy as a function of inverse filling (dashed curve) and is expected to win for fillings less than about $\frac{1}{6}$. (After Manoharan et al. [28].)

ballistic CF transport in nanostructures. The results of most of these experiments are in general agreement with each other and with the CF picture although some inconsistencies exist. Among the unresolved topics are the CF effective mass and the degree of CF spin polarization.

\subsection{The Wigner crystal state}

As mentioned earlier, the ground state of a 2DES at very high magnetic fields, i.e., in the limit of $\nu \rightarrow 0$ is expected to be a Wigner crystal (WC), namely, an ordered array of electrons (see Fig. 2, top right panel). A few words regarding the competition between the FQHE and the WC are therefore in order. It turns out that the Laughlin FQHE liquid states at $\nu=p / q$ are particularly robust and have ground state energies which are lower than the WC state energy, at least for $\nu>\frac{1}{5}^{1}$. This is illustrated in Fig. 9 where the estimated energies are plotted as a function of $\nu$ (for details of estimations see Ref. 28). The downward "cusps" in energy reflect the incompressibility of the FQHE states and the presence of energy gaps which are proportional to the discontinuties in the derivative of energy vs $\nu$. Also shown schematically in Fig. 9 (dashed curve) is the expected dependence of the WC ground state energy on $\nu$. Theoretical calculations predict that, in an ideal $2 \mathrm{D}$ system, the WC should be the ground state for $\nu$ smaller than about $\frac{1}{6}$. It is evident from Fig. 9 that while at $\nu=\frac{1}{5}$ the FQHE can be the ground state, the WC state may win as

\footnotetext{
${ }^{1}$ This is not necessarily true for the higher Landau levels (see Section 3.6)
} 


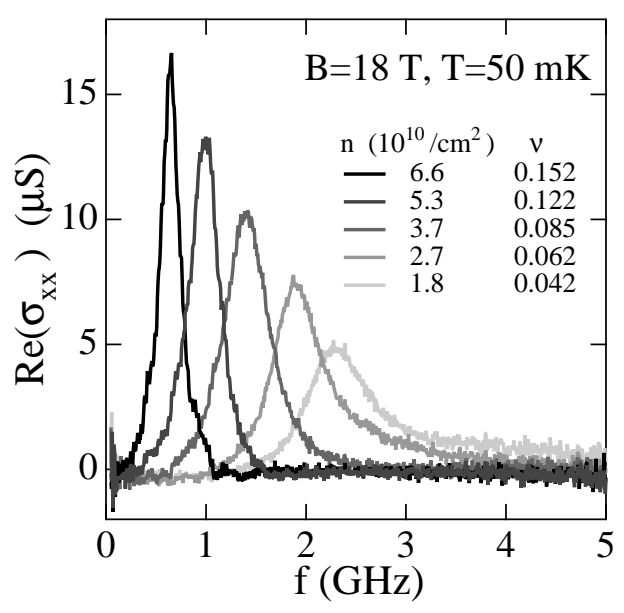

Figure 10: Resonance spectra, showing the real part of the diagonal conductivity as a function of frequency for a high mobility GaAs 2DES . Data are shown for a number of 2D densities at a fixed field of $18 \mathrm{~T}$. (After Ye et al. [29.)

the filling deviates slightly from $\frac{1}{5}$. It is possible therefore to have a WC which is reentrant around a FQHE liquid state.

The above picture has been used to rationalize the general current belief that the insulating phase (IP) observed around the $\nu=\frac{1}{5}$ FQHE in very high quality GaAs/AlGaAs 2DESs (e.g., see Fig. 1) is the signature of a pinned WC state. The solid is presumably "pinned" by the disorder potential, and can be made to slide if a sufficiently large electric field is applied. Such depinning would result in a nonlinear current-voltage characteristic and various resonances, consistent with numerous measurements. The magnetic-field-induced WC problem in 2D systems has been studied extensively since the late 1980's [20, 21].

High frequency measurements of the conductivity have proven to be a valuable tool in the regime of small filling factors where the WC phase is presumably dominant [29]. An example is shown in Fig. 10 for a very high quality GaAs 2DES. The data show a rather sharp conductivity resonance at a frequency whose position and characteristics, e.g., its behavior with temperature, density, and magnetic field, are consistent with the pinning mode of a magnetic-field-induced WC. Moreover, analysis of the resonance data based on pinned WC models yields domain sizes that are many times the inter electron spacing [29].

It is worth mentioning that strikingly similar reentrant IPs have been observed in other high-quality GaAs 2D carrier systems, such as in 2D hole systems or in bilayer systems of either electrons or holes. As summarized in Fig. 11, however, the IPs in these systems occur at much larger fillings. In a dilute 2D hole system, e.g., an IP reentrant around the $\nu=\frac{1}{3}$ is observed [20], while interacting bilayer electron or hole systems with appropriate parameters show such phases at even higher fillings (see Section 4.4). These observations can be qualitatively understood in terms of the profound effect of Landau level mixing 


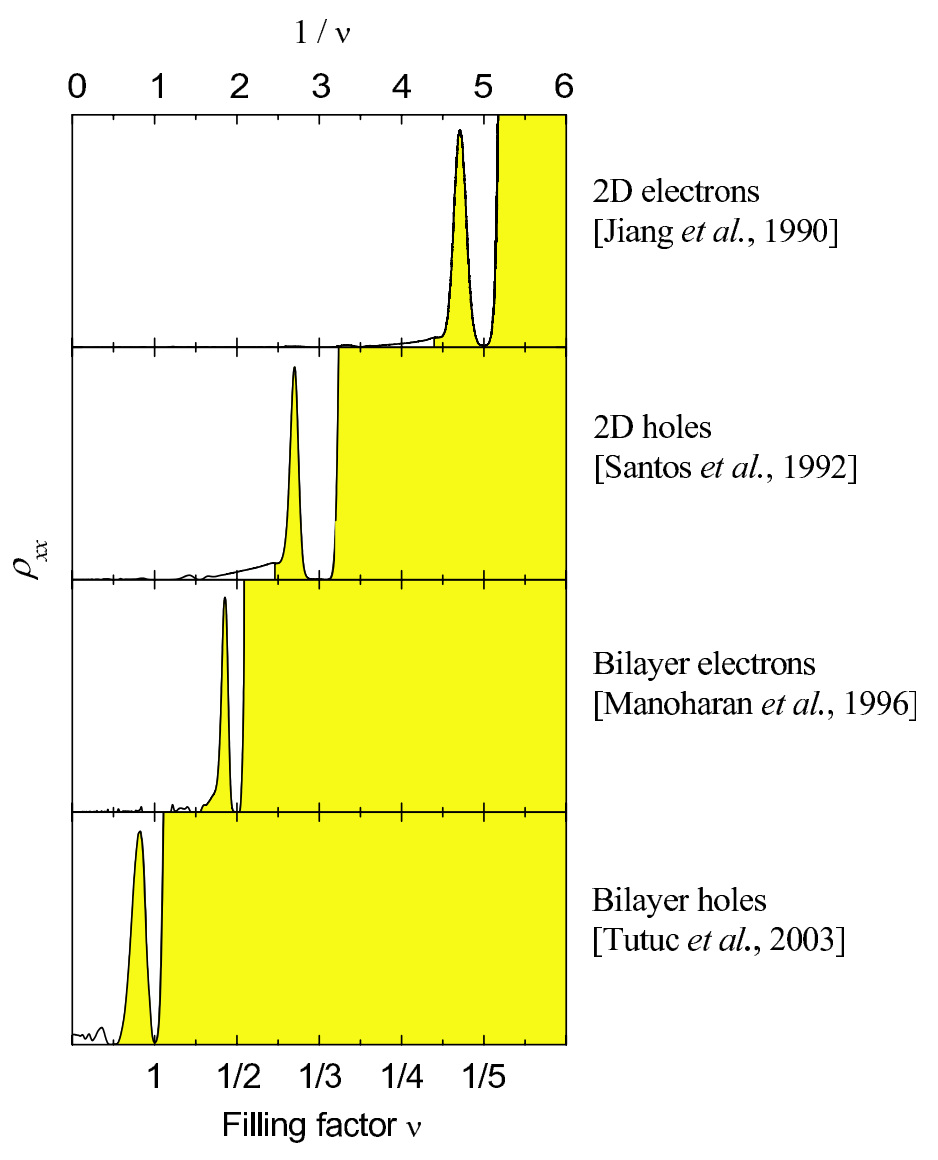

Figure 11: Summary of reentrant insulating phases (shaded areas) observed at low temperatures in various GaAs 2D carrier systems. (After Jiang et al. [30; Santos et al. [31; Manoharan et al. [32; Tutuc et al. 33.)

(effective diluteness) in the case of the 2D holes and of the interlayer interaction in the case of the bilayer systems, both of which significantly modify the ground-state energies of the FQHE and WC states of the system and shift the liquid-to-solid transition to larger $\nu[20$. These results are very suggestive and provide further credibility to the interpretation of the IP as a pinned WC.

\subsection{Ferromagnetic state at $\nu=1$ and Skyrmions}

For 2DESs in GaAs, while the IQHE at even $\nu$ arises from the single-particle energy gaps separating the LLs, the spin splitting of these levels leads to IQHE at odd $\nu$. The electronelectron interaction and in particular the exchange energy, however, play a dominant role for odd- $\nu$ IQHE and often lead to a substantially larger QHE energy gap than expected from the bare effective $g$-factor $\left(g^{*} \simeq-0.44\right)$ for GaAs [34]. In fact, according to theory [35, the odd- $\nu$ IQHE states should exist even in the limit of zero Zeeman energy $\left(g^{*} \rightarrow 0\right)$; there 
should be a spontaneous ferromagnetic order with a spin polarized 2DES ground state.

Perhaps even more interesting are the predicted excitations of these ferromagnetic states: provided that $g^{*}$ is sufficiently small, the charged excitations of the system are finite-size "Skyrmions", termed so after the work of Skyrme in 1958 on baryons in nuclear matter 36, rather than single spin flips. Skyrmions are spin textures, smooth distortions of the spin field involving several spin flips (see Fig. 2, bottom center panel) [35, 37]. The spin and size of the Skyrmions are determined by the competition between the Zeeman and the exchange energies: a large ratio of the exchange energy over the Zeeman energy would favor large-size Skyrmions over single spin flips as the (exchange) energy gained by the near parallelism of the spins would outweigh the (Zeeman) energy cost of the extra spin flips. Skyrmions are relevant at $\nu=1$ (at finite $T$ ) and near $\nu=1$ where the 2DES is not fully spin polarized.

Clear experimental evidence for finite-size Skyrmions was provided by the pioneering nuclear magnetic resonance measurements of Barrett et al. [38. On either side of $\nu=1$, they observed a rapid drop of the Knight-shift of the ${ }^{71} \mathrm{Ga}$ nuclei which are in contact with or are near the 2DES. Associating this Knight-shift with the spin polarization of the 2DES, they deduced that the charged excitations of the $\nu=1$ QHE carry large $(\simeq 4)$ spins 38 . This work has been followed by numerous experimental and theoretical studies, providing additional credence to the Skyrmionic picture near $\nu=1$ [37.

Implied by the Knight-shift data [37, 38, is a strong coupling of the nuclear and 2DES spin systems near $\nu=1$ where Skyrmions are present. Here I would like to discuss some 2DES heat capacity $(C)$ data near $\nu=1$ at very low $T$ [39] which dramatically manifest the consequences of this Skyrmion-induced coupling. Moreover, a remarkably sharp peak observed in $C$ vs $T$ is suggestive of a phase transition in the electronic system, possibly signaling a crystallization of the Skyrmions at very low $T$.

Bayot et al. [39] succeeded in measuring $C$ vs $B$ and $T$ in a GaAs/AlGaAs multiplequantum-well sample in the QHE regime down to $T \simeq 25 \mathrm{mK}$ (Fig. 12). Their $C$ vs $B$ data is striking in that at high $B$ (near $\nu=1) C$ becomes many orders of magnitude larger than its low $B$ value. While the low $B$ data can be understood based on the 2DES electronic heat capacity and its oscillating density of states at the Fermi energy, the high $B$ data near $\nu=1$ are unexpected and cannot be accounted for based on the thermodynamic properties of the 2DES alone. Both the very large magnitude of $C$ and the $T^{-2}$ dependence of $C$ at high $T$ (dashed line in Fig. 12) hint at the nuclear Schottky effect 39]. Utilizing this clue, Bayot et al. were able to semi-quantitatively explain the magnitude and the dependence of $C$ on $B$ and $T$ (for $T>0.1 \mathrm{~K}$ ) based on a simple Schottky model for the nuclear spins of the Ga and As atoms in the quantum wells. Implicit in this interpretation of course is a coupling between the nuclear spins and the lattice; this coupling is assumed to be provided by the Skyrmions.

Figure 12 reveals yet another intriguing feature of the heat capacity data: in a small range of $\nu$ near 0.8 (and also near 1.2), $C$ vs $T$ exhibits a very sharp peak at a temperature $T_{c}$ which sensitively depends on $\nu$ [39]. The Schottky model, however, predicts a smooth maximum in $C$ at $T \sim \delta / 2 k_{B} \simeq 2 \mathrm{mK}$ for $B \simeq 7 \mathrm{~T}$, the field position of $\nu \simeq 0.8$, and cannot explain the sharp peak observed at $T_{c} \sim 35 \mathrm{mK}$ ( $\delta$ is the nuclear spin splitting). This peak is possibly a signature of the expected Skyrmion crystallization and the associated magnetic 


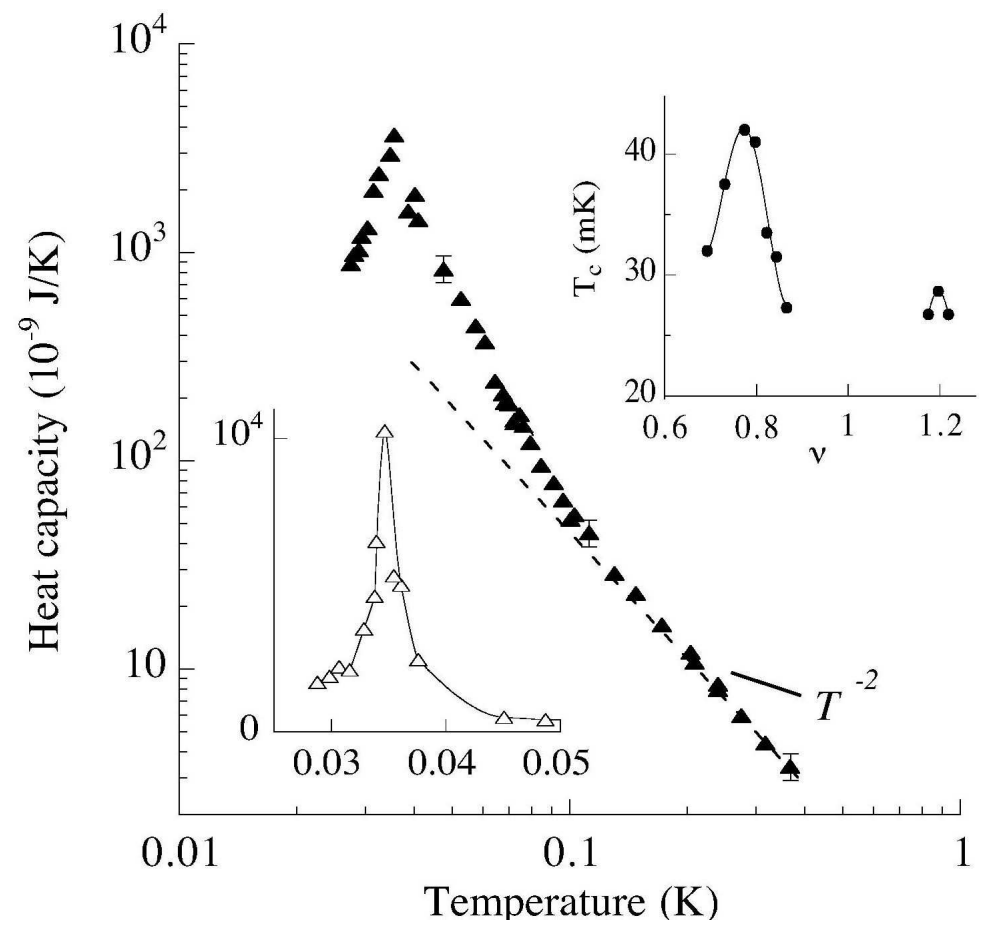

Figure 12: Temperature dependence of the heat capacity near filling factor one $(\nu=0.81)$ is shown in the main figure in a log-log plot for a multiple-quantum-well GaAs sample. The dashed line shows the $T^{-2}$ dependence expected for the Schottky model. The lower inset shows a linear plot of the heat capacity vs temperature at $\nu=0.85$. The temperature $T_{c}$, at which the heat capacity exhibits the sharp peak depends on the filling factor as shown in the upper inset. (After Bayot et al. [39].)

ordering near $\nu=1$ [39, 40]. Such crystallization has indeed been proposed theoretically [40] although the details of the Skyrmion liquid-solid transition and, in particular, how it would affect the coupling to the nuclear spin system are unknown. One feature of the data that qualitatively agrees with the Skyrmion crystallization is worth emphasizing. As shown in the upper inset in Fig. 12, the observed $T_{c}$ decreases rapidly as $\nu$ deviates from 0.8 or 1.2 [39]; this is consistent with the expectation that as the Skyrmion density decreases, the Skyrme crystal melting temperature should decrease. Qualitatively consistent with the heat capacity data and interpretation are the results of a recent study of the nuclear magnetic resonance at very low temperatures in a high-quality, single-layer, GaAs 2DES [4].

\subsection{Excited Landau levels: competition between uniform- and modulated- density many-body states}

The physics of $2 \mathrm{D}$ carrier systems in the second and higher LLs is particularly delicate 42 , 43. The electron wavefunction in these excited levels has a larger extent and also possesses one or more nodes. These combine to modify the (exchange-correlation) interaction effects, 


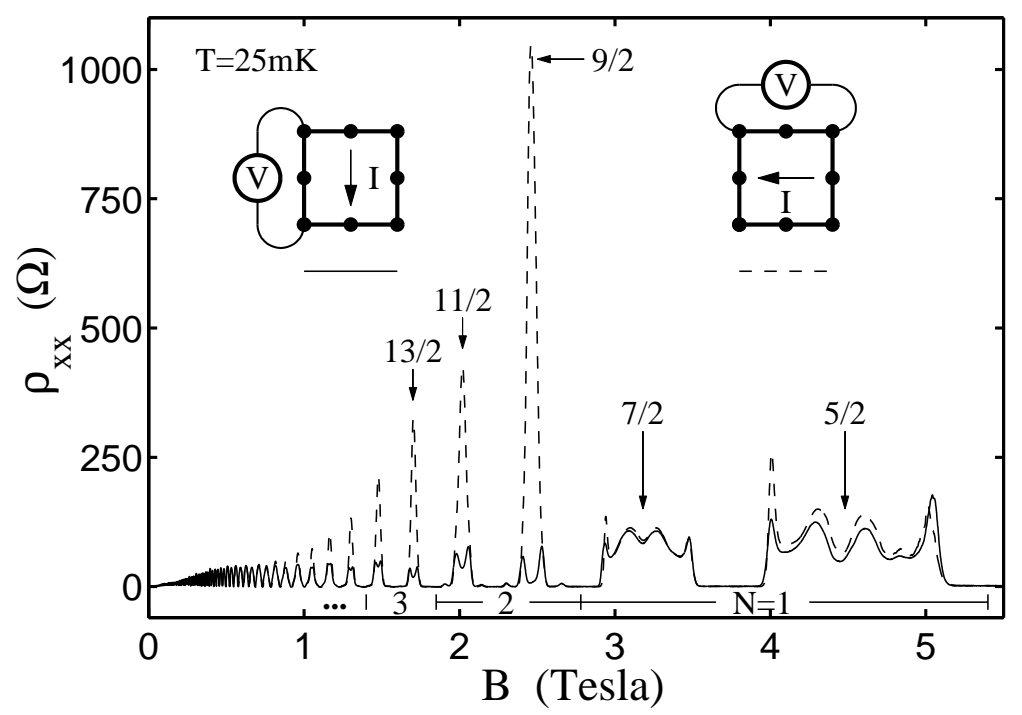

Figure 13: Longitudinal magnetoresistance data for a high mobility GaAs 2DES, revealing extreme anisotropy of $\rho_{x x}$ at half-integer fillings $\nu \geq \frac{9}{2}$. The two traces correspond to two different orientations of the current through the sample (inest). (After Lilly et al. 44].)

and lead to a very close competition between the uniform-density, liquid states and densitymodulated phases such as Wigner crystal or charge-density-waves. Since these states are bunched together in filling factor (e.g., $2<\nu<3$ ) and energy, their observation requires the highest quality samples and lowest temperatures. Thanks to the availability of ultra-high mobility GaAs 2DESs, it has become possible in recent years to experimentally explore the high LLs in detail and indeed many new phases have emerged.

Figure 13 highlights one of the novel features observed in high quality 2D carrier systems at high LLs 42, 44, 45, 46]: the longitudinal resistivity exhibits a very large in-plane anisotropy at half-integer fillings $\nu \geq \frac{9}{2}$. The anisotropy develops only at very low temperatures and can be as large as a factor of 10 in Hall bar geometry samples (the anisotropy is exaggerated in van der Pauw geometry samples [47]). Its origin is believed to be the formation of interaction-induced "stripe" phases at these half-filled LLs (see Ref. 42 for a brief review). At $\nu=\frac{9}{2}$, e.g., the interaction leads to a phase separation of the 2DES into stripes with $\nu=4$ and 5 fillings (see Fig. 2, bottom right panel). Such striped, charge-densitywave states were in fact theoretically predicted in Hartree-Fock calculations [48, 49, 50. The resistivity is small (easy axis) when the current is passed parallel to the stripes and large (hard axis) when the direction of the current is perpendicular to the stripes. There is experimental evidence that the direction of stripes can be rotated by adding a parallel component to the magnetic field [51, 52. There is, however, no clear picture yet as to what determines the direction of the stripes in a purely perpendicular field [42, 53, 54,

Figure 14 provides yet another example of magnetotransport data 43 at $T=9 \mathrm{mK}$ of an extremely high-quality GaAs 2DES with a mobility of $3.1 \times 10^{7} \mathrm{~cm}^{2} /$ Vs. Here data are shown in the filling factor range $2<\nu<3$. Several FQHE states are marked by arrows, 


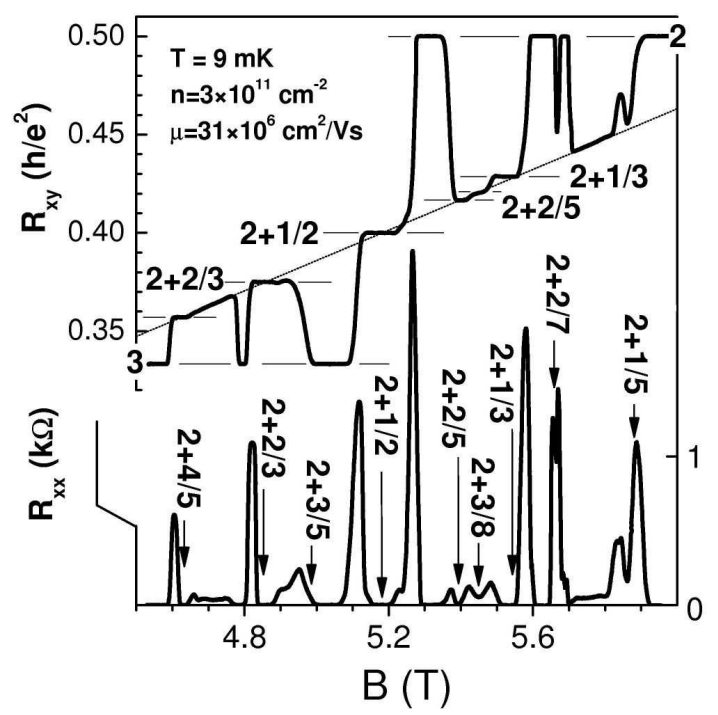

Figure 14: Magnetotransport coefficients in the excited Landau level (between $\nu=2$ and $\nu=3$ ) at a very low temperature of $9 \mathrm{mK}$ for a very high quality GaAs 2DES. The data reveal the complex and delicate competition between FQHE states (marked by vertical arrows) whose Hall resistance $R_{x y}$ is quantized at values on the classical Hall line, and reentrant IQHE states whose $R_{x y}$ is quantized at either two or three times $h / e^{2}$. (After Xia et al. [43.)

including one at $\nu=\frac{5}{2}$. This incompressible liquid state is very special since, unlike all the other QHE states in a single-layer 2DES, it occurs at an even-denominator filling. Recall that the odd-denominator rule is linked to the requirement that the many-body FQHE wavefunction be anti-symmetric (see equation 1). Although the $\frac{5}{2}$ FQHE state was first observed quite some time ago [55, its origin is not yet fully understood (for recent results see Refs. 56-58). In particular, it is still debated whether it is a spin-polarized or unpolarized state [57, and also whether a pairing of electrons or composite Fermions is responsible for the formation of this state. The latest experimental data suggest that there is a Fermi sea of composite Fermions at high temperatures and that it is the pairing of these at the lowest temperatures that leads to the $\frac{5}{2}$ FQHE [58].

Another noteworthy feature of the data of Fig. 14 is that in certain ranges of magnetic field, e.g., between 5.0 and $5.1 \mathrm{~T}, R_{x x}$ vanishes while the Hall resistance $R_{x y}$ attains a quantized value equal to the neighboring integer QHE plateaus (3h/e $e^{2}$ in this example) 43. 59. These so called reentrant QHE states exhibit remarkable non-linear current-voltage characteristics and narrow-band noise [60], as well as pronounced resonances at microwave frequencies 61, 62]. The data all signal that these are non-uniform density, many-body states and, together with theoretical results, suggest the presence of exotic pinned Wigner crystal and "bubble" phases (a Wigner crystal phase with more than one electron in the unit cell). 


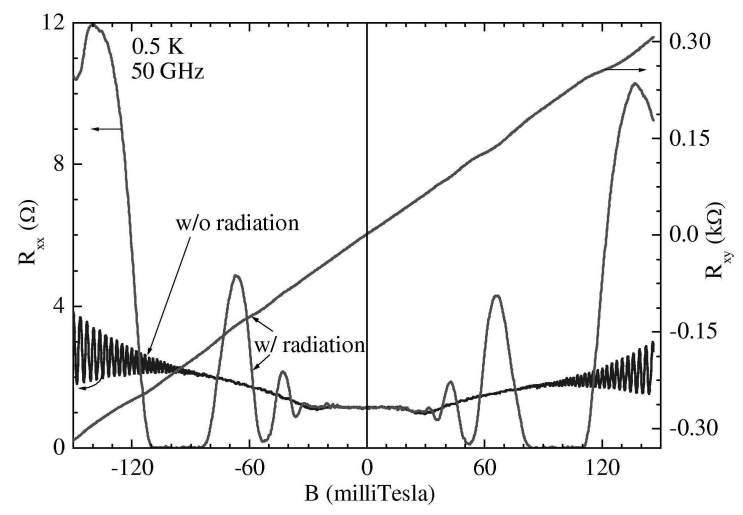

Figure 15: Magnetotransport coefficients of a high mobility GaAs 2DES under $50 \mathrm{GHz}$ incident radiation. Under radiation, at low magnetic fields, the longitudinal resistance $R_{x x}$ develops deep minima that approach zero at low temperatures but no plateaus are formed in the Hall resistance $R_{x y}$. (After Mani et al. 65].)

\subsection{Radiation-induced "zero-resistance" states at low fields}

This is one of the latest developments in the physics of 2DESs in a perpendicular magnetic field 63, 64, 65, 66. Figure 15 shows the basic observation 65. When a very high quality 2DES is irradiated with microwaves $(f=50 \mathrm{GHz}$ for Fig. 15 data), its longitudinal resistance develops deep oscillations at very low magnetic fields, corresponding to $\nu>50$. The resistance minima get stronger and become vanishingly small as the temperature is lowered toward absolute zero. Similar to the Shubnikov de Haas effect, these oscillations are periodic in $B^{-1}$, but the positions of the minima are not determined by the chemical potential and the filling factor, but rather by the ratio of the microwave and cyclotron frequencies. This observation implies that these oscillations have a semi-classical origin. Moreover, as seen in Fig. 15, there are no plateaus in the Hall resistance so the phenomenon is distinct from the QHE.

The observation of these so-called radiation-induced "zero-resistance" states has generated an enormous number of theoretical papers (see 67] for a partial list of some of the theory papers). While it is premature to say that the problem is entirely understood, the plausible picture that appears to be emerging is that this is not a new collective phenomenon, but rather a manifestation of non-equilibrium dynamics under the influence of microwave radiation. In particular, a simple model, based on the effect of microwave radiation on impurity scattering can explain the period and phase of the resistance oscillations 67, 68, 69, 70. The model in fact predicts negative resistance minima, i.e., the amplitude of the oscillations should grow with the intensity of the microwaves so that, at sufficiently large intensity, the resistance should become negative in certain ranges of the magnetic field. Experimentally, however, the resistance minima appear to saturate as they approach zero (Fig. 15). A theoretical explanation for this saturation has been proposed based on current instabilities associated with the negative resistance [71, 72. The explanation asserts that a 
uniform current is unstable if $R_{x x} \rightarrow 0$, and that the sample breaks into domains, in some of which the current flows opposite to the applied current. Such domains are yet to be detected in measurements.

\section{Correlated bilayer electron states}

\subsection{Overview}

The introduction of an additional degree of freedom can have a profound effect on the many-body ground states of the 2DES at high $B$ [3]. For example, the addition of a spin degree of freedom stabilizes particular spin-unpolarized FQHE observed at lower $B$ [74, while substantially increasing the layer thickness (thus introducing an additional spatial degree of freedom) leads to a weakening and eventual collapse of the FQHE [23, 24]. On the other hand, adding a layer degree of freedom can lead to novel correlated states some of which have no counterpart in single-layer 2DESs. For example, when two electron layers are brought to close proximity so that the interlayer and intralayer Coulomb interactions are comparable, new QHE states ensue. Such "two-component" QHE states have a generalized Laughlin wavefunction of the form [75, 76, 77]:

$$
\Psi_{m m l}^{\nu} \sim \prod_{i, j}\left(u_{i}-u_{j}\right)^{m} \prod_{i, j}\left(w_{i}-w_{j}\right)^{m} \prod_{i, j}\left(u_{i}-w_{j}\right)^{l} \times \exp \left(-\sum_{i} \frac{\left|u_{i}\right|^{2}}{4 l_{B}^{2}}-\sum_{i} \frac{\left|w_{i}\right|^{2}}{4 l_{B}^{2}}\right)
$$

where $u_{i}$ and $w_{i}$ denote the complex $2 \mathrm{D}$ coordinates of the electrons in the two layers. The integer exponents $m$ and $l$ determine the intralayer and interlayer correlations, respectively, and the total filling factor for the $\Psi_{m m l}^{\nu}$ state is $\nu=2 /(m+l)$.

A measure of how close one needs to bring two electron layers for novel bilayer phenomena to occur is the ratio of the intralayer and interlayer Coulomb interaction, $\left(e^{2} / 4 \pi \varepsilon l_{B}\right) /\left(e^{2} / 4 \pi \varepsilon d\right)=$ $d / l_{B}$, where $d$ is the interlayer distance and $l_{B}=(\hbar / e B)^{1 / 2}$ is the magnetic length. Now the two-component QHE states described by $\Psi_{m m l}^{\nu}$ come in two classes. For large $d / l_{B}$, the system behaves as two independent layers in parallel, each with half the total density. The FQHE states in this regime therefore have even numerator and odd denominator. An example is the $\Psi_{330}^{2 / 3}$ state which has a total filling of $\frac{2}{3}$ ( $\frac{1}{3}$ filling in each layer). Note that the exponent $l=0$ means that there is no interlayer correlation. For small enough $d / l_{B}$, on the other hand, fundamentally new QHE states with strong interlayer correlation are possible. Two such states that have been observed so far are the $\Psi_{331}^{1 / 2}$ and $\Psi_{111}^{1}$ states at $\nu=\frac{1}{2}$ and at $\nu=1$, respectively. I will briefly present these states in the next two sections and then discuss another phenomenon, namely the presence of insulating phases at high filling factors, that suggests the formation of bilayer Wigner crystal states.

Before presenting data, it is worth noting that a high quality, GaAs/AlGaAs-based bilayer electron (or hole) system can be realized in two distinct types of structures. One is a double quantum well (QW) structure where the electrons are confined to two GaAs wells that are separated by an AlGaAs barrier (Fig. 16, left side). A second, less intuitive structure is a single, wide GaAs QW of width $\sim 100 \mathrm{~nm}$. At low density the electrons occupy 


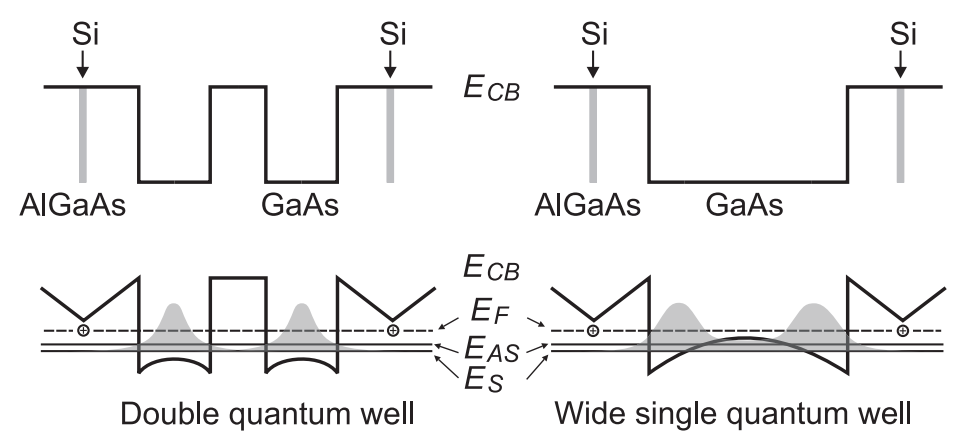

Figure 16: Schematic figure showing the formation of a bilayer electron system in either a double quantum well (left) or a wide quantum well (right). In each case, the conduction band edge is shown before (top) and after (bottom) the charge transfer from the dopants to the quantum well(s). Note that in the wide quantum well case, the "barrier" between the layers results from the (self-consistent) electrostatic repulsion between the electrons themselves.

the lowest electric subband and have a single-layer-like (but rather "thick" in the growth direction) charge distribution. As more electrons are added to the well, their electrostatic repulsion forces them to pile up near the well's sides and the resulting electron charge distribution appears increasingly bilayer-like (Fig. 16, right side). The wide QW system is particularly interesting since both the inter-layer tunneling and, to some degree, the distance between the layers, can be tuned in situ by adding or removing electrons from the QW (via the application of front- and back-sides) [32, 78, 79]. This means that in a wide QW of fixed width, the system can be essentially tuned from a bilayer to a (thick) single-layer by decreasing the density. This evolution with density plays a decisive role in the properties of the correlated electron states in this system [32, 78, 79].

\subsection{Even-denominator quantum Hall states in bilayer systems}

Figure 17 exhibits data for an electron system in a $75 \mathrm{~nm}$-wide, single GaAs quantum well. As seen in the main panel, there is a well-developed FQHE state at the even-denominator filling $\nu=\frac{1}{2}$ [80]. A similar FQHE state has also been observed in GaAs double QW samples 81]. The inset to Fig. 17 reveals a FQHE state at yet another even-denominator filling $\nu=\frac{3}{2}$ [82. Neither of these even-denominator states has a counterpart in standard 2DESs in single-heterostructures. I emphasize that the fillings marked in Fig. 17 are the total fillings of the bilayer system; e.g., $\nu=\frac{1}{2}$ corresponds to $\frac{1}{4}$ filling for each layer.

The FQHE states at $\nu=\frac{1}{2}$ observed in the double QW and the wide single QW structures are believed to be signatures of the $\Psi_{331}^{1 / 2}$ state. (The state at $\nu=\frac{3}{2}$ can be understood as the hole conjugate of the $\frac{1}{2}$ state.) In the double QW system, the FQHE state at $\nu=\frac{1}{2}$ is observed for $d / l_{B} \simeq 2$, consistent with theoretical expectations for the $\Psi_{331}$ state. In the wide QW system, on the other hand, the $\frac{1}{2}$ state is stable at much larger $d / l_{B}$ values $(\sim 6)$. This is likely because the larger thickness of the electron layers in the 


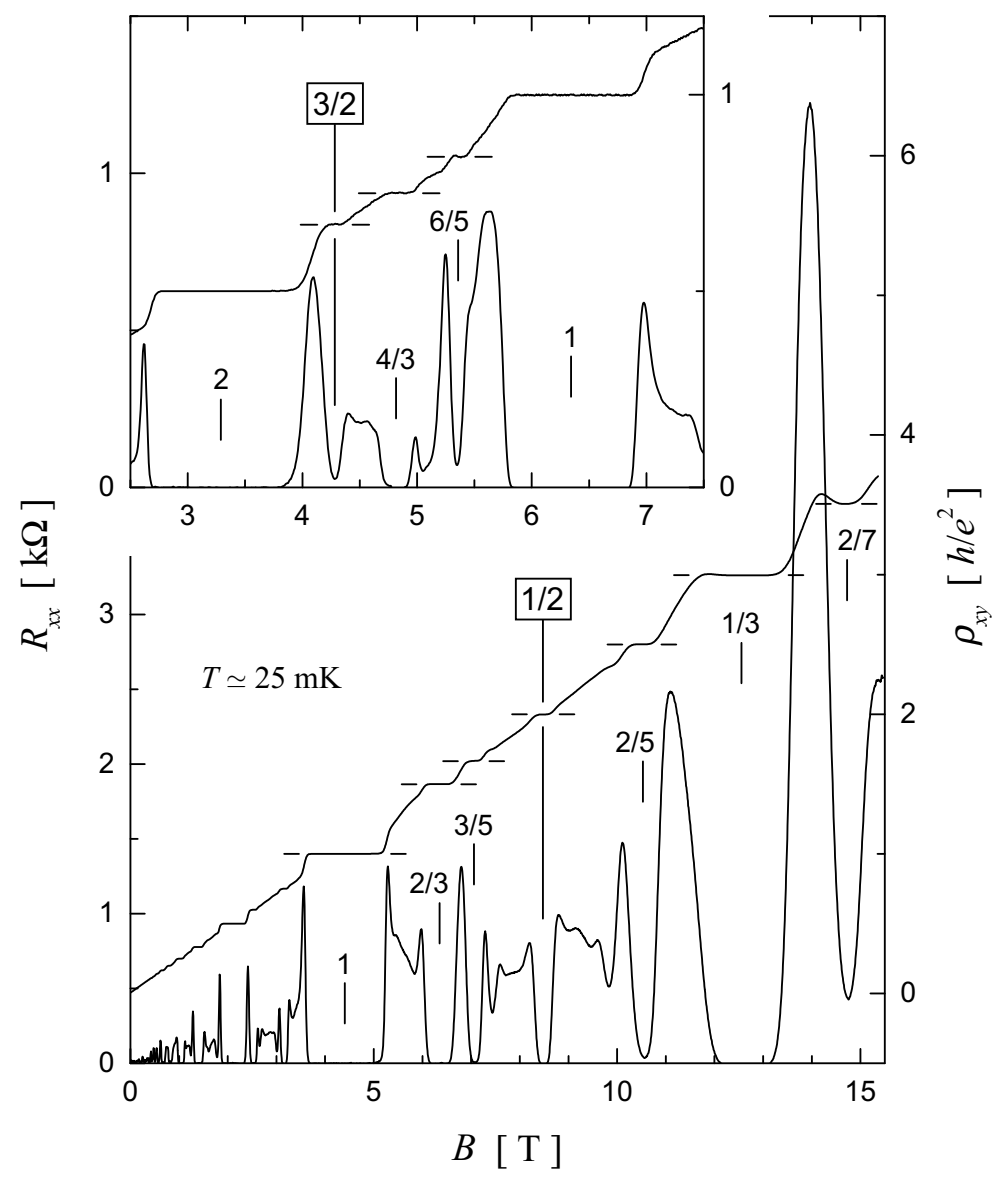

Figure 17: Magnetotransport data, taken at $T \simeq 30 \mathrm{mK}$, for a $75 \mathrm{~nm}$-wide, single GaAs quantum well with $n=1.03 \times 10^{11} \mathrm{~cm}^{-2}$ (main figure) and $n=1.55 \times 10^{11} \mathrm{~cm}^{-2}$ (inset), showing well-developed even-denominator FQHE states at $\nu=\frac{1}{2}$ and $\frac{3}{2}$, respectively. These unique FQHE states are stabilized by both interlayer and intralayer correlations. (After Suen et al. [80.)

wide QW leads to a softening of the intralayer interaction in this system [82].

\subsection{The bilayer QHE at $\nu=1$ : electron-hole pairing and Bose-Einstein condensation}

In closely spaced bilayer systems, the interlayer and intralayer interactions can also lead to the $\Psi_{111}$ QHE state at total filling factor $\nu=1$ [83, 84, 85, 86]. As can be seen from equation (2), the exponents of the three terms in the $\Psi_{111}$ wavefunction are all equal, meaning that this state enjoys similar interlayer and intralayer correlations. As a result, this is a very special state: it possesses unique, interlayer phase coherence that leads to exotic properties such as electron-hole pairing and Bose-Einstein condensation 84, 86. To understand the physics of this peculiar QHE state note that, at $\nu=1$, the carriers in each layer occupy 


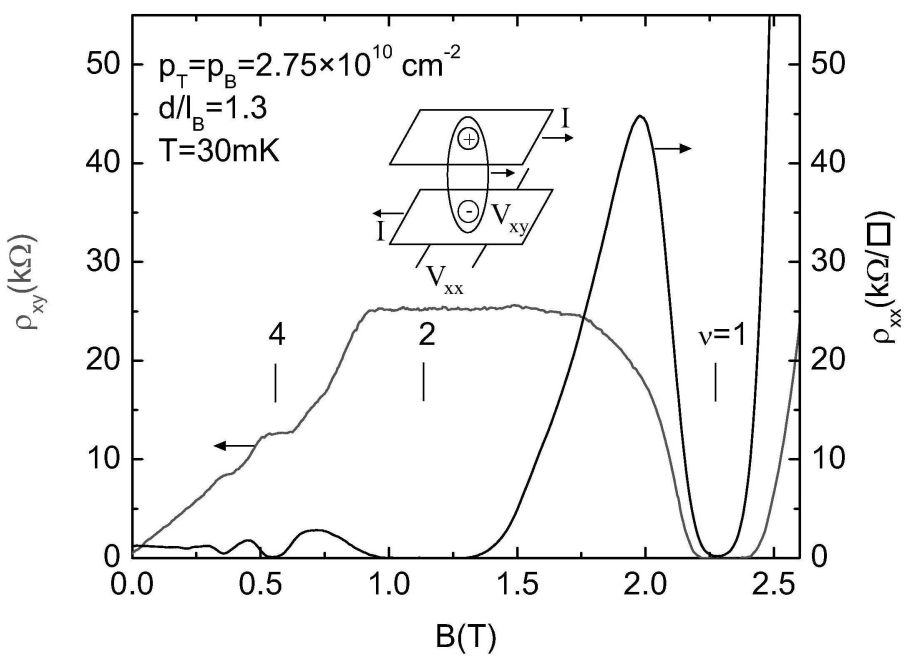

Figure 18: Magnetotransport coefficients of a bilayer 2D hole system in the counterflow geometry (inset) where the current is passed through the two layers in opposite directions. Both the longitudinal and the Hall resistivity vanish in the $\nu=1 \mathrm{QHE}$ state. The vanishing of the Hall resistivity signals the formation of bilayer electron-hole pairs (excitons), shown schematically in the inset. (After Tutuc et al. 90.)

exactly half of the available Landau orbits, leaving the other half vacant. Under proper circumstances, namely at low temperatures and when $d / l_{B} \simeq 1$, the carriers in one layer "pair" with the vacancies in the opposite layer and form neutral excitons. These excitons in turn can condense into a superfluid state below a critical temperature. Alternatively, one can ascribe the layer degree of freedom in this system to a pseudo-spin. The system's ground state is then a QHE ferromagnet where all the pseudo-spins align in the same direction.

Experimental data have already shown that the bilayer $\nu=1$ QHE exhibits novel phenomena such as Josephson-like interlayer tunneling [87] and quantized Hall drag [88]. Here I present an example of the fascinating phenomenon recently observed in this system, namely electron-hole pairing and signatures of superfluidity in the "counterflow" transport configuration [89, 90, 91. Figure 18 shows such counterflow data for a bilayer GaAs hole system [90; qualitatively similar data have also been reported for bilayer electron systems 89, 91. In the counterflow geometry, equal currents are passed in opposite directions in the two, independently contacted layers (see inset to Fig. 18). At $\nu=1$, both the longitudinal and the Hall counterflow resistances tend to vanish in the limit of zero temperature. The vanishing of the Hall resistivity is especially important since it directly demonstrates that the counterflow current is carried by neutral particles, that is, by particle-vacancy pairs which have zero electric charge and therefore experience no Lorentz force. The vanishing of the longitudinal resistivity in the limit of zero temperature implies that the ground state of the system is an excitonic Bose-Einstein condensate (superfluid). It is worth emphasizing that the longitudinal conductivity $\sigma_{x x}$, determined by inverting the measured longitudinal 
and Hall resistivities, diverges as $T \rightarrow 0$. This is consistent with a superfluid ground state, and opposite to what is observed in the usual QHE where the finite $\rho_{x y}$ leads to a vanishing $\sigma_{x x}$ in the limit of zero temperature. So far, however, a transition to the superfluid state at finite temperature has not been observed experimentally, possibly because of residual sample disorder.

\subsection{Insulating phases in bilayer systems: evidence for a bilayer Wigner cr.}

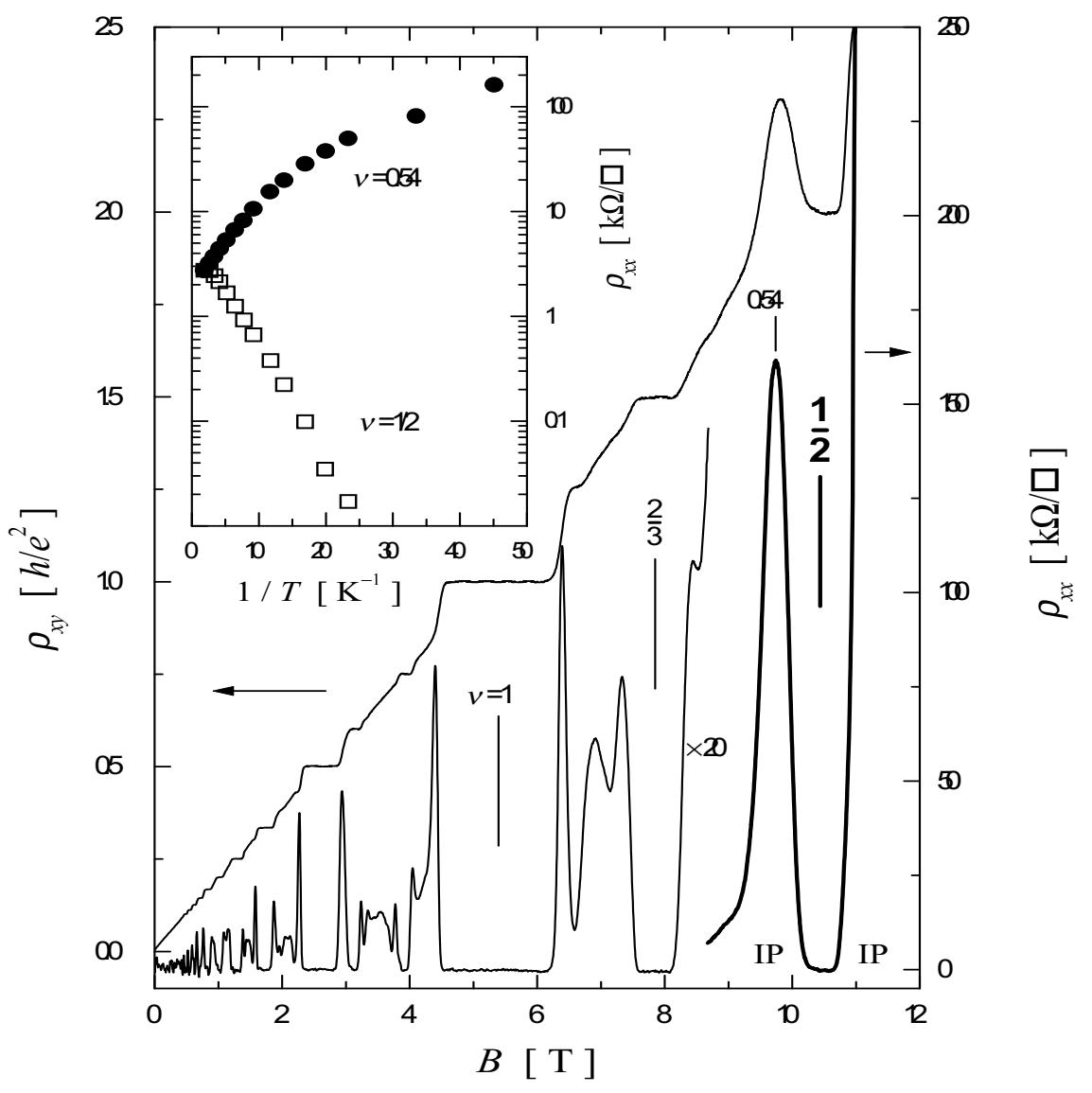

Figure 19: Data for the same sample of Fig. 17 but with $n=1.26 \times 10^{11} \mathrm{~cm}^{-2}$. Here we an insulating phase reentrant around the $\nu=\frac{1}{2} \mathrm{FQHE}$ state is observed. The inset shows the temperature dependence of resistivity: at $\nu=\frac{1}{2}, \rho_{x x}$ vanishes as $T \rightarrow 0$ indicative of a FQHE state while at slightly higher and lower $\nu, \rho_{x x}$ shows an insulating behavior as it diverges with decreasing T. (After Manoharan et al. [32.)

Figure 19 reveals yet another interesting observation in interacting bilayer systems, namely, the development of insulating phases (IPs) that are reentrant around FQHE states at rather high fillings. In Fig. 19, transport data on the same wide QW as in Fig. 17 but 
at a higher density $\left(n \simeq 1.26 \times 10^{11} \mathrm{~cm}^{-2}\right)$ show IPs reentrant around the $\nu=\frac{1}{2} \mathrm{FQHE}$ state. Note that $\nu=\frac{1}{2}$ means a layer filling of $\frac{1}{4}$, i.e., a filling which is larger than $\frac{1}{5}$ where an IP is observed in single-layer, GaAs 2DESs (see Fig. 11 in Section 3.4). Presumably, the interlayer interaction is leading to a bilayer, pinned Wigner crystal. The evolution of this insulating phase as a function of total bilayer density and layer density imbalance was found to be consistent with this conjecture [20, 32, 79].

Experimental data on interacting, GaAs, bilayer hole systems further corroborate this conclusion. In the case of holes, an IP reentrant around $\nu=1$ is observed (Fig. 11) 33, 92]. Here the IP is seen around the bilayer $\left(\Psi_{111}\right)$ QHE state, i.e., near a layer filling of $\frac{1}{2}$ which is larger than $\nu=\frac{1}{3}$ where the IP in a single-layer 2D hole system occurs (see Fig. 11), again suggesting that interlayer interaction has shifted the onset of the Wigner crystal formation to higher fillings.

\section{$5 \quad$ Summary and future perspectives}

In this article I have attempted to provide a glimpse of the exciting phenomena that $2 \mathrm{D}$ carrier systems in a perpendicular magnetic field have revealed over the last 25 years or so. With improvements in sample quality, it is more than likely that new surprises continue to emerge. This is particularly true for the excited Landau levels (Section 3.6) where there is fierce competition between various uniform- and modulated-density many body states. Higher quality samples and lower temperatures are key to the observation and further understanding of such states.

A second area where more surprises are likely to emerge is in studies of $2 \mathrm{D}$ carrier systems in novel structures and materials. Examples are the bilayer or, more generally, multicomponent carrier systems. Such systems possess an additional degree of freedom, e.g., layer, spin, or valley degree of freedom, and this can lead to phenomena that at times have no counterpart in one-component systems (see Section 4 for examples). Recently there has also been progress in the fabrication of high-quality multi-valley systems, including $2 \mathrm{D}$ electrons confined to AlAs 93] or Si 94 quantum wells, and the new systems have indeed revealed intriguing FQHE phenomena stemming from their multiple valley occupation. An example is shown in Fig. 20 for an AlAs quantum well where the 2D electrons occupy two conduction band valleys [93. A developing FQHE state is observed at a very high filling of $\nu=\frac{11}{3}$, and there are also hints of FQHE states emerging at higher fillings (e.g., $\nu=\frac{13}{3}, \frac{14}{3}$, and even $\frac{17}{3}$ when the sample is tilted with respect to the direction of magnetic field [93]). Fractional QHE states at such high fillings are either absent or rarely seen in standard, GaAs 2DESs of even the highest quality, and are likely a result of the multi-valley electron occupation.

In closing, I would like to emphasize that the focus of this paper has been transport measurements on 2D carrier systems in a perpendicular magnetic field. Much can be learned about the physics of $2 \mathrm{D}$ systems by either adding an in-plane component of the magnetic field, or applying a purely in-plane field. For example, at appropriate tilt angles, one can bring the Landau levels of opposite spin into coincidence and study phenomena such as quantum Hall ferromagnetism [95, 96]. Or by applying a purely in-plane field, one can 


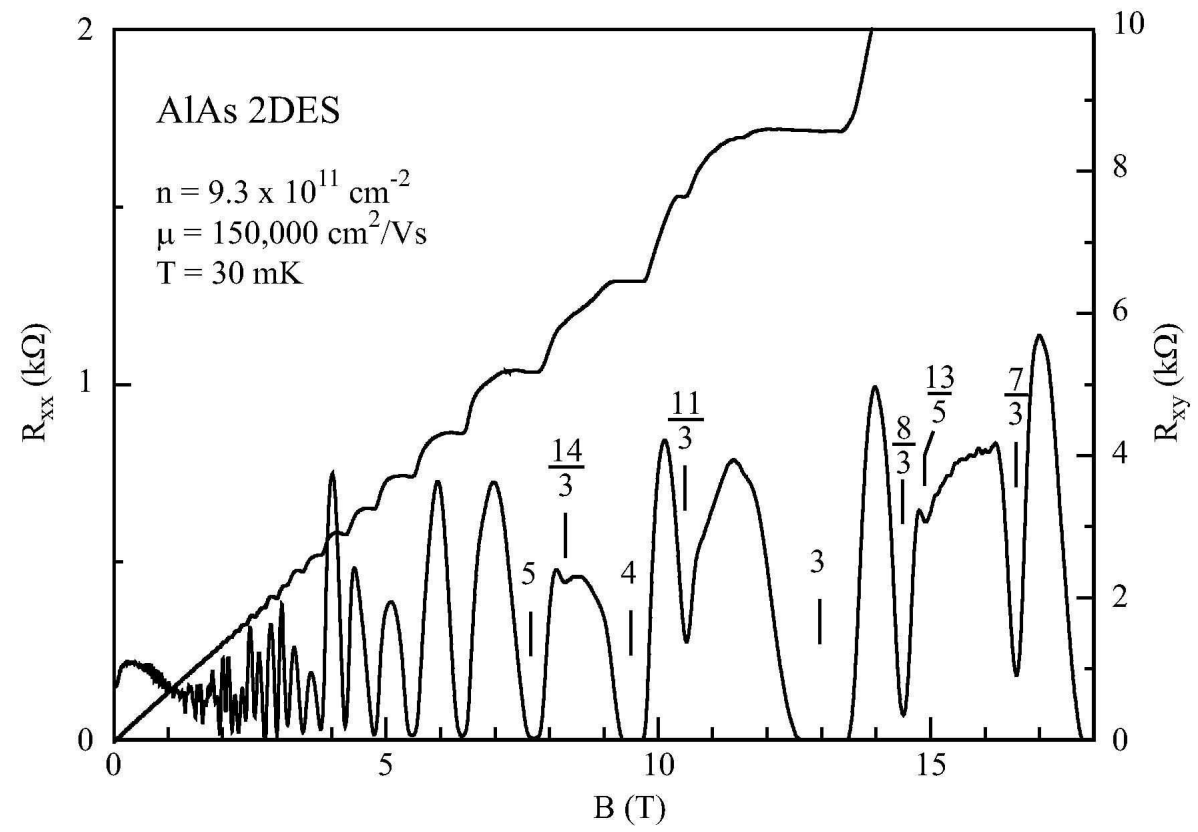

Figure 20: Magnetotransport data for 2D electrons occupying two conduction band valleys in an AlAs quantum well. The data exhibit developing FQHE states at unusually large fractional fillings such as $\nu=\frac{11}{3}$. (After De Poortere et al. [93].)

study the spin polarization of $2 \mathrm{D}$ systems and determine their spin susceptibility (see, e.g., Ref. 97 and references therein).

Finally, magnetic fields are also invaluable tools in studying systems with dimensions lower than two. Examples include quantum wire (1D) and dot (0D) systems. In such systems, the magnetic field can couple to the spin of the carriers and, for appropriate geometries and parameters, also to their orbital motion. These systems are the subjects of intensive current research, thanks to their exciting basic properties as well as potential device applications [5].

\section{Acknowledgments}

I thank all the colleagues in the field of $2 \mathrm{D}$ carrier systems in a magnetic field for years of hard work, illuminating discussions, fruitful collaborations, and exciting competition! I apologize for not giving adequate credit to everyone's work and for being biased toward work done in my lab with which I am most familiar. Many thanks to L.W. Engel, S.M. Girvin, A.H. MacDonald, D.C. Tsui, and E. Tutuc, for a critical reading of this article. I am indebted to E.P. De Poortere, L.W. Engel, V.J. Goldman, O. Gunawan, M.P. Lilly, R. Mani, H.C. Manoharan, S. Melinte, W. Pan, Y.P. Shkolnikov, and E. Tutuc for providing me with figures, including some unpublished ones. I also thank Y.P. Shkolnikov, O. Gunawan, and 
S. Braude for help preparing the manuscript. The work at Princeton University has been supported primarily by the National Science Foundation and the Department of Energy.

\section{$7 \quad$ References}

\section{References}

[1] T. Sajoto, Y.P. Li, L.W. Engel, D.C. Tsui, and M. Shayegan, Phys. Rev. Lett. 70, 2321 (1993).

[2] K. von Klitzing, G. Dorda, and M. Pepper, Phys. Rev. Lett. 45, 494 (1980).

[3] D.C. Tsui, H.L. Stormer, and A.C. Gossard, Phys. Rev. Lett. 48, 1559 (1982).

[4] R.B. Laughlin, Phys. Rev. Lett. 50, 1395 (1983).

[5] Many of the reports on the physics of 2D systems can be found in the proceedings of the international conferences on the Electronic Properties of Two-dimensional Systems (EP2DS), which are held every two years. For recent proceedings, see: Surf. Sci. 305 (1994); Surf. Sci. 361/362 (1996); Physica B 249-251 (1998); Physica E 6 (2000); Physica E 12 (2002); Physica E 22 (2004).

[6] R.E. Prange and S.M. Girvin, eds., The Quantum Hall Effect, 2nd ed., (Springer-Verlag, New York, 1990).

[7] T. Chakraborty and P. Pietilainen, The Quantum Hall Effects: Fractional and Integral, (Springer-Verlag, Berlin, 1995).

[8] S. Das Sarma and A. Pinczuk, eds., Perspectives in Quantum Hall Effects, (Wiley, New York, 1997).

[9] For a simple and useful text on semiconductor heterostructures, see J.H. Davies, The Physics of Low-Dimensional Semiconductors, (Cambridge University Press, Cambridge, 1998). Other useful books and review articles are: G. Bastard, Wave Mechanics Applied to Semiconductor Heterostructures, (Halsted Press, New York, 1988); C. Weisbuch and B. Vinter, Quantum Semiconductor Structures, (Academic Press, New York, 1991); M.J. Kelly, Low-Dimensional Semiconductors, Materials, Physics, Technology, Devices, (Clarendon Press, Oxford, 1995).

[10] For a comprehensive review of the $2 \mathrm{D}$ physics in the pre-QHE era see T. Ando, A.B. Fowler, and F. Stern, Rev. Mod. Phys. 54, 437 (1982).

[11] H.L. Stormer, R. Dingle, A.C. Gossard, W. Wiegmann, and M.D. Sturge, Solid State Commun. 29, 705 (1979).

[12] A.Y. Cho, Materials Research Society (MRS) Bulletin 20, 21 (1995). 
[13] M. Shayegan, V.J. Goldman, M. Santos, T. Sajoto, L. Engel, and D.C. Tsui, Appl. Phys. Lett. 53, 2080 (1988); M. Shayegan, V.G. Goldman,C. Jiang, T. Sajoto, and M. Santos, Appl. Phys. Lett. 52, 1086 (1988).

[14] L.N. Pfeiffer, K.W. West, H.L. Stormer, and K.W. Baldwin, Appl. Phys. Lett. 55, 1888 (1989).

[15] For a 30 nm-wide, GaAs quantum well, grown by L.N. Pfeiffer and K.W. West at Lucent Technologies, a mobility of $3.1 \times 10^{7} \mathrm{~cm}^{2} / \mathrm{Vs}$ at a density of $3 \times 10^{11} \mathrm{~cm}^{-2}$ has been reported (data of Fig. 14, Section 3.6).

[16] T. Sajoto, Y.W. Suen, L.W. Engel, M.B. Santos, and M. Shayegan, Phys. Rev. B 41, 8449 (1990).

[17] B.E. Kane, L.N. Pfeiffer, K.W. West, and C.K. Harnett, Appl. Phys. Lett. 63, 2132 (1993).

[18] F. Stern, Appl. Phys. Lett. 43, 974 (1983); A. Gold, Phys. Rev. B 44, 8818 (1991).

[19] C.C. Grimes and G. Adams, Phys. Rev. Lett. 42, 795 (1979).

[20] For a review of the developments in the magnetic-field-induced Wigner crystal states of 2D systems up to 1996 see articles by M. Shayegan (experiments) and H.A. Fertig (theory) in Ref. 8.

[21] W. Pan, H.L. Stormer, D.C. Tsui, L.N. Pfeiffer, K.W. Baldwin, and K.W. West, Phys. Rev. Lett. 88, 176802 (2002).

[22] R.L. Willett, H.L. Stormer, D.C. Tsui, A.C. Gossard, and J.H. English, Phys. Rev. B 37, 8476 (1988).

[23] M. Shayegan, J. Jo, Y.W. Suen, M. Santos, and V.J. Goldman, Phys. Rev. Lett. 65, 2916 (1990).

[24] S. He, F.C. Zhang, X.C. Xie, and S. Das Sarma, Phys. Rev. B 42, 11376 (1990).

[25] R. de-Picciotto, M. Reznikov, M. Heiblum, V. Umansky, G. Bunin, and D. Mahalu, Nature 389, 162 (1997); L. Saminadayar, D.C. Glattli, Y. Jin, and B. Etienne, Phys. Rev. Lett. 79, 2526 (1997).

[26] For a review of composite Fermions and the FQHE, see theory articles by J.K. Jain and by B.I. Halperin in Ref. 8. Also included in Ref. 8 is a comprehensive review, by H.L. Stormer and D.C. Tsui, of the experimental results supporting the CF picture. For another illuminating review, see R.L. Willett, Adv. Phys. 46, 447 (1997).

[27] V.J. Goldman, B. Su, and J.K. Jain, Phys. Rev. Lett. 72, 2065 (1994).

[28] H.C. Manoharan, Y.W. Suen, T.S. Lay, M.B. Santos, and M. Shayegan, Phys. Rev. Lett. 79, 2722 (1997); H.C. Manoharan, Ph.D. Thesis, Princeton University, 1998. 
[29] P.D. Ye, L.W. Engel, D.C. Tsui, R.M. Lewis, L.N. Pfeiffer, and K.W. West, Phys. Rev. Lett. 89, 176802 (2002).

[30] H.W. Jiang, R.L. Willett, H.L. Stormer, D.C. Tsui, L.N. Pfeiffer, and K.W. West, Phys. Rev. Lett. 65, 633 (1990).

[31] M.B. Santos, Y.W. Suen, M. Shayegan, Y.P. Li, L.W. Engel, and D.C. Tsui, Phys. Rev. Lett. 68, 1188 (1992).

[32] H.C. Manoharan, Y.W. Suen, M.B. Santos, and M. Shayegan, Phys. Rev. Lett. 77, $1813(1996)$.

[33] E. Tutuc, S. Melinte, E.P. DePoortere, R. Pillarisetty, and M. Shayegan, Phys. Rev. Lett. 91, 076802 (2003).

[34] R.J. Nicholas, R.J. Haug, K. von Klitzing, and G. Weimann, Phys. Rev. B 37, 1294 (1988).

[35] S.L. Sondhi, A. Karlhede, S.A. Kivelson, and E.H., Rezayi, Phys. Rev. B 47, 16419 (1993).

[36] T.H.R. Skyrme, Proc. R. Soc. London, Ser. A 247, 260 (1958).

[37] See, e.g., S.M. Girvin's Les Houches 1998 notes in Topologial Aspects of Low Dimensional Systems, edited by A. Comtet, T. Jolicoer, S. Ouvry, and F. David, (Springer, Berlin, 1999), pp. 53-175.

[38] S.E. Barrett, G. Dabbagh, L.N. Pfeiffer, K.W. West, and R. Tycko, Phys. Rev. Lett. 74, 5112 (1995).

[39] V. Bayot, E. Grivei, M.B. Santos, and M. Shayegan, Phys. Rev. Lett. 76, 4584 (1996); V. Bayot, E. Grivei, J.-M. Beuken, S. Melinte, and M. Shayegan, Phys. Rev. Lett. 79, $1718(1997)$.

[40] R. Côté, A.H. MacDonald, L. Brey, H.A. Fertig, S.M. Girvin, and H.T.C. Stoof, Phys. Rev. Lett. 78, 4825 (1997).

[41] G. Gervais, H.L Stormer, D.C. Tsui, P.L. Kuhns, W.G. Moulton, A.P. Reyes, L.N. Pfeiffer, K.W. Baldwin, and K.W. West, cond-mat/0412140.

[42] For a brief review, see J.P. Eisenstein, Solid State Commun. 117, 123 (2001).

[43] J.S. Xia, W. Pan, C.L. Vicenta, E.D. Adams, N.S. Sullivan, H.L. Stormer, D.C. Tsui, L.N. Pfeiffer, K.W. Baldwin, and K.W. West, Phys. Rev. Lett. 93, 176809 (2004).

[44] M.P. Lilly, K.B. Cooper, J.P. Eisenstein, L.N. Pfeiffer, and K.W. West, Phys. Rev. Lett. 82, 394 (1999).

[45] R.R. Du, D.C. Tsui, H.L. Stormer, L.N. Pfeiffer, and K.W. West, Solid State Commun. 109, 389 (1999). 
[46] M. Shayegan, H.C. Manoharan, S.J. Papadakis, and E.P. DePoortere, Physica (Amsterdam) 6E, 40 (2000).

[47] S. Simon, Phys. Rev. Lett. 83, 4223 (1999).

[48] A.A. Kulakov, M.M. Fogler, and B.I. Shklovskii, Phys. Rev. Lett. 76, 449 (1996).

[49] M.M. Fogler, A.A. Kulakov, and B.I. Shklovskii, Phys. Rev. B 54, 1853 (1996).

[50] R. Moessner and J.T. Chalker, Phys. Rev. B 54, 5006 (1996).

[51] W. Pan, R.R. Du, H.L. Stormer, D.C. Tsui, L.N. Pfeiffer, K.W. Baldwin, and K.W. West, Phys. Rev. Lett. 83, 820 (1999).

[52] M.P. Lilly, K.B. Cooper, J.P. Eisenstein, L.N. Pfeiffer, and K.W. West, Phys. Rev. Lett. 83, 824 (1999).

[53] R.L. Willett, J.W.P. Hsu, D. Natelson, K.W. West, and L.N. Pfeiffer, Phys. Rev. Lett. 87, 126803 (2001).

[54] J. Zhu, W. Pan, H.L. Stormer, L.N. Pfeiffer, and K.W. West, Phys. Rev. Lett. 88, 116803 (2002).

[55] R.L. Willett, J.P. Eisenstein, H.L. Stormer, D.C. Tsui, A.C. Gossard, and J.H. English, Phys. Rev. Lett. 59, 1776 (1987); F.D.M. Haldane and E.H. Rezayi, Phys. Rev. Lett. 60, 956 (1988).

[56] W. Pan, J. S. Xia, V. Shvarts, D.E. Adams, H.L. Stormer, D.C. Tsui, L.N. Pfeiffer, K.W. Baldwin, and K. West, Phys. Rev. Lett. 83, 3530 (1999).

[57] W. Pan, H.L. Stormer, D.C. Tsui, L.N. Pfeiffer, K.W. Baldwin, and K. West, Solid State Commun. 119, 641 (2001).

[58] R.L. Willett, K.W. West, and L.N. Pfeiffer, Phys. Rev. Lett. 88, 066801 (2002).

[59] J.P. Eisenstein, K.B. Cooper, L.N. Pfeiffer, and K.W. West, Phys. Rev. Lett. 88, 076801 (2002).

[60] K.B. Cooper, J.P. Eisenstein, L.N. Pfeiffer, and K.W. West, Phys. Rev. Lett. 90, 226803 (2003).

[61] R.M. Lewis, P.D. Ye, L.W. Engel, D.C. Tsui, L.N. Pfeiffer, and K.W. West, Phys. Rev. Lett. 89, 136804 (2002).

[62] Y. Chen, R.M. Lewis, L.W. Engel, D.C. Tsui, P.D. Ye, L.N. Pfeiffer, and K.W. West, Phys. Rev. Lett. 91, 016801 (2003).

[63] M.A. Zudov, R.R. Du, J.A. Simmons, and J.L. Renon, Phys. Rev. B 64, 201311(R) (2001). 
[64] P.D. Ye, L.W. Engel, D.C. Tsui, J.A. Simmons, J.R. Wendt, G.A. Vawter, J.L. Reno, Appl. Phys. Lett. 79, 2193 (2001).

[65] R. Mani, J.H. Smet, K. Von Klitzing, V. Narayanmurti, W.B. Johnson, and V. Umansky, Nature (London) 420, 646 (2002).

[66] M.A. Zudov, R.R. Du, L.N. Pfeiffer, and K.W. West, Phys. Rev. Lett. 90, 046807 (2003).

[67] A.C. Durst, S. Sachdev, N. Read, and S.M. Girvin, Physica E 25, 198 (2004).

[68] A.C. Durst, S. Sachdev, N. Read, and S.M. Girvin, Phys. Rev. Lett. 91, 086803 (2003).

[69] V.I. Ryzhii, Fiz. Tverd. Tela (Leningrad) 11, 2577 (1969) [Sov. Phys. Solid State 11, 2078 (1970)].

[70] V.I. Ryzhii, R.A. Suris, and B.S. Shchamkhalova, Fiz. Tekh. Fiz. Tekh. Poluprovodn. 20, 2078 (1986) [Sov. Phys. Semicond. 20, 1289 (1986)].

[71] A.V. Andreev, I.L. Aleiner, and A.J. Millis, Phys. Rev. Lett. 91, 056803 (2003).

[72] M.G. Vavilov and I.L. Aleiner, Phys. Rev. B 69, 035303 (2004).

[73] For reviews, see articles by J.P. Eisenstein and by S.M. Girvin and A.H. MacDonald in Ref. 8.

[74] R.G. Clark, S.R. Haynes, A.M. Suckling, J.R. Mallett, P.A. Wright, J.J. Harris, and C.T. Foxon, Phys. Rev. Lett. 62, 1536 (1989); J.P. Eisenstein, H.L. Stormer, L.N. Pfeiffer, and K.W. West, ibid 1540 (1989).

[75] B.I. Halperin, Helv. Phys. Acta. 56, 75 (1983).

[76] D. Yoshioka, A.H. MacDonald, and S.M. Girvin, Phys. Rev. B 39, 1932 (1989).

[77] A.H. MacDonald, Surf. Sci. 229, 1 (1990).

[78] Y.W. Suen, J. Jo, M. Santos, L.W. Engel, S.W. Hwang, and M. Shayegan, Phys. Rev. $B$ 44, 5947 (1991); Y.W. Suen, Ph.D. Thesis, Princeton University, 1994.

[79] For a review, see M. Shayegan's Les Houches 1998 notes in Topological Aspects of Low Dimensional Systems, edited by A. Comtet, T. Jolicoer, S. Ouvry, and F. David, (Springer, Berlin, 1999), pp. 1-51.

[80] Y.W. Suen, L.W. Engel, M.B. Santos, M. Shayegan, and D.C. Tsui, Phys. Rev. Lett. 68, 1379 (1992).

[81] J.P. Eisenstein, G.S. Boebinger, L.N. Pfeiffer, K.W. West, and S. He, Phys. Rev. Lett. 68, 1383 (1992). 
[82] Y.W. Suen, H.C. Manoharan, X. Ying, M.B. Santos, and M. Shayegan, Phys. Rev. Lett. 72, 3405 (1994).

[83] T. Chakraborty and P. Pietilainen, Phys. Rev. Lett. 59, 2784 (1987); H.A. Fertig, Phys. Rev. B 40, 1087 (1989).

[84] X.G. Wen and A. Zee, Phys. Rev. Lett. 69, 1811 (1992); F. Ezawa and A. Iwazaki, Int. J. Mod. Phys. B 6, 3205 (1992).

[85] S.Q. Murphy, J.P. Eisenstein, G.S. Boebinger, L.N. Pfeiffer, and K.W. West, Phys. Rev. Lett. 72, 728 (1994).

[86] K. Moon, H. Mori, K. Yang, S.M. Girvin, A.H. MacDonald, A.H. Zheng, D. Yoshioka, and S.C. Zhang, Phys. Rev. B 51, 5138 (1995).

[87] I.B. Spielman, J.P. Eisenstein, L.N. Pfeiffer, and K.W. West, Phys. Rev. Lett. 84, 5808 (2000).

[88] M. Kellogg, I.B. Spielman, J.P. Eisenstein, L.N. Pfeiffer, and K.W. West, Phys. Rev. Lett. 88, 126804 (2002).

[89] M. Kellogg, J.P. Eisenstein, L.N. Pfeiffer, and K.W. West, Phys. Rev. Lett. 93, 036801 (2004).

[90] E. Tutuc, M. Shayegan, and D. Huse, Phys. Rev. Lett. 93, 036802 (2004).

[91] R.D. Wiersma, J.G.S. Lok, S. Kraus, W. Dietsche, K. von Kliezing, D. Schuh, M. Bichler, H. P. Tranitz, and W. Wegscheider, Phys. Rev. Lett. 93, 266805 (2004).

[92] S. Faniel, E. Tutuc, E.P. De Poortere, C. Gustin, A. Vlad, S. Melinte, M. Shayegan, and V. Bayot, Phys. Rev. Lett. 94, 046802 (2005).

[93] E.P. De Poortere, Y. Shkolnikov, E. Tutuc, S.J. Papadakis, M. Shayegan, E. Plam, and T. Murphy, Appl. Phys. Lett. 80, 1583 (2002); E.P. De Poortere, Ph.D. Thesis, Princeton University, 2003.

[94] K. Lai, W. Pan, D.C. Tsui, S. Lyon, M. Mühlberger, and F. Schäffler, Phys. Rev. Lett. 93, 156805 (2004).

[95] T. Jungwirth and A.H. MacDonald, Phys. Rev. B 63, 035305 (2000).

[96] E.P. De Poortere, E. Tutuc, S.J. Papadakis, and M. Shayegan, Science 290, 1546 (2000).

[97] Y.P. Shkolnikov, K. Vakili, E.P. De Poortere, and M. Shayegan, Phys. Rev. Lett. 92, 246804 (2004). 